\title{
EXACT AND ANALYTIC-NUMERICAL SOLUTIONS OF STRONGLY COUPLED MIXED DIFFUSION PROBLEMS
}

\author{
L. JÓDAR ${ }^{1}$, E. NAVARRO ${ }^{1}$ AND J. A. MARTIN ${ }^{2}$ \\ ${ }^{1}$ Departamento de Matemática Aplicada, Universidad Politécnica de Valencia, \\ P.O. Box 22012, Valencia, Spain (ljodar@mat.upv.es) \\ ${ }^{2}$ Departamento de Análisis Matemático y Matemática Aplicada, \\ Universidad de Alicante, Ap. Correos 99, E-03080 Alicante, \\ Spain (jose.martin@ua.es)
}

(Received 16 March 1998)

\begin{abstract}
This paper deals with the construction of exact and analytical-numerical solutions with $a$ priori error bounds for systems of the type $u_{t}=A u_{x x}, A_{1} u(0, t)+B_{1} u_{x}(0, t)=0, A_{2} u(1, t)+B_{2} u_{x}(1, t)=$ $0,0<x<1, t>0, u(x, 0)=f(x)$, where $A_{1}, A_{2}, B_{1}$ and $B_{2}$ are matrices for which no simultaneous diagonalizable hypothesis is assumed, and $A$ is a positive stable matrix. Given an admissible error $\varepsilon$ and a bounded subdomain $D$, an approximate solution whose error with respect to an exact series solution is less than $\varepsilon$ uniformly in $D$ is constructed.
\end{abstract}

Keywords: coupled diffusion problem; coupled boundary conditions; vector boundary-value differential system; analytic-numerical solution; Moore-Penrose pseudoinverse

AMS 1991 Mathematics subject classification: Primary 35C10; 35M10

Secondary $15 \mathrm{~A} 24$

\section{Introduction and preliminaries}

Coupled partial differential systems with coupled boundary-value conditions are frequent in quantum mechanical scattering problems $[\mathbf{1}, \mathbf{1 9}, \mathbf{2 7}]$, chemical physics $[\mathbf{1 6}, \mathbf{1 7}, \mathbf{2 2}]$, thermoelastoplastic modelling [13], coupled diffusion problems $[\mathbf{8}, \mathbf{2 0}, \mathbf{2 9}]$, and other fields. In this paper we consider systems of the type

$$
\begin{gathered}
u_{t}(x, t)-A u_{x x}(x, t)=0, \quad 0<x<1, \quad t>0, \\
A_{1} u(0, t)+B_{1} u_{x}(0, t)=0, \quad t>0, \\
A_{2} u(1, t)+B_{2} u_{x}(1, t)=0, \quad t>0, \\
u(x, 0)=f(x), \quad 0 \leqslant x \leqslant 1,
\end{gathered}
$$

where the unknown $u=\left(u_{1}, u_{2}, \ldots, u_{m}\right)^{\mathrm{T}}$ and $f=\left(f_{1}, f_{2}, \ldots, f_{m}\right)^{\mathrm{T}}$ are $m$-dimensional vectors, $A_{i}, B_{i}, i=1,2$ are $m \times m$ complex matrices, elements of $\mathbb{C}^{m \times m}$, and $A$ is a positive stable matrix

$$
\operatorname{Re}(z)>0 \text { for all eigenvalues } z \text { of } A \text {. }
$$


We assume that

$$
\left.\begin{array}{l}
\text { The block matrix }\left(\begin{array}{ll}
A_{1} & B_{1} \\
A_{2} & B_{2}
\end{array}\right) \text { is invertible and } \\
\text { not all its blocks } A_{1}, A_{2}, B_{1}, B_{2} \text { are singular. }
\end{array}\right\}
$$

Conditions on the function $f(x)$ and on the matrix coefficients will be determined in order to guarantee the existence of a series solution of the problem, as well as the construction of analytic-numerical finite-sum approximations with a prefixed accuracy in a bounded subdomain. Mixed problems of the above type, but with Dirichlet conditions $u(0, t)=0$, $u(1, t)=0$ instead of equations (1.2) and (1.3), have been treated in $[\mathbf{1 5}, \mathbf{2 3}]$.

The organization of the paper is as follows. In $\S 2$, the vector eigenvalue differential problem

$$
\left.\begin{array}{c}
X^{\prime \prime}(x)+\lambda^{2} X(x)=0, \quad 0<x<1, \quad \lambda \geqslant 0, \\
A^{j} X(0)+B_{1} A^{j} X^{\prime}(0)=0, \\
A_{2} A^{j} X(1)+B_{2} A^{j} X^{\prime}(1)=0, \\
0 \leqslant j \leqslant p-1, \quad p \geqslant 1,
\end{array}\right\}
$$

is studied. Sufficient conditions for the existence of eigenvalues are given. Using a separation-of-variables technique, an exact series solution of problems (1.1)-(1.4) is constructed in $\S 3$. In $\S 4$, a procedure for the construction of a finite-sum approximation with a prefixed accuracy is given, by truncation of the exact infinite-series solution and appropriate approximations of the eigenvalues.

Throughout this paper, the set of all the eigenvalues of a matrix $C$ in $\mathbb{C}^{m \times m}$ is denoted by $\sigma(C)$ and its 2-norm denoted by $\|C\|$ is defined by $[\mathbf{1 1}, \mathbf{p .} 56]$

$$
\|C\|=\sup _{z \neq 0} \frac{\|C z\|_{2}}{\|z\|_{2}}
$$

where, for a vector $y$ in $\mathbb{C}^{m},\|y\|_{2}$ denotes the usual Euclidean norm of $y$. By $[11$, p. 556], it follows that

$$
\left\|\mathrm{e}^{t C}\right\| \leqslant \mathrm{e}^{t \alpha(C)} \sum_{k=0}^{m-1} \frac{\|\sqrt{m} C\|^{k} t^{k}}{k !}, \quad t \geqslant 0,
$$

where $\alpha(C)=\max \{\operatorname{Re}(w) ; w \in \sigma(C)\}$. The conjugate transpose of $C$ is denoted by $C^{*}$. If $B$ is a matrix in $\mathbb{C}^{n \times m}$, we denote by $B^{\dagger}$ its Moore-Penrose pseudoinverse. An account of examples, properties and applications of this concept may be found in $[\mathbf{6}]$ and $[\mathbf{2 6}]$, and $\mathrm{B}^{\dagger}$ can be efficiently computed with the MATLAB package. The kernel of $B$, denoted by ker $B$, coincides with the image of the matrix $I-B^{\dagger} B$, denoted by $\operatorname{Im}\left(I-B^{\dagger} B\right)$, see [6]. We say that a subspace $E$ of $\mathbb{C}^{m}$ is invariant by the matrix $A \in \mathbb{C}^{m \times m}$ if $A(E) \subset E$. Hence, property $A(\operatorname{ker} G) \subset \operatorname{ker} G$ is equivalent to the condition $G A\left(I-G^{\dagger} G\right)=0$. We conclude this section with an algebraic result that will play an important role in the following. 
Lemma 1.1. Let $M$ and $N$ be matrices in $\mathbb{C}^{m \times m}$, then

$\operatorname{ker} M \cap \operatorname{ker} N=\operatorname{Im}\left\{\left(I-M^{\dagger} M\right)\left\{I-\left[N\left(I-M^{\dagger} M\right)\right]^{\dagger}\left[N\left(I-M^{\dagger} M\right)\right]\right\}\right\}$.

Proof. If $v \in \operatorname{ker} M \cap \operatorname{ker} N$, then $M v=0$, and, by Theorem 2.3 .2 of [26, p. 24], $v=\operatorname{Im}\left(I-M^{\dagger} M\right) d$, where $d$ is an arbitrary vector in $\mathbb{C}^{m}$. Hence

$$
v \in \operatorname{Im}\left(I-M^{\dagger} M\right) \supset \operatorname{Im}\left\{\left(I-M^{\dagger} M\right)\left\{I-\left[N\left(I-M^{\dagger} M\right)\right]^{\dagger}\left[N\left(I-M^{\dagger} M\right)\right]\right\}\right\} .
$$

Conversely, let $v \in \operatorname{Im}\left\{\left(I-M^{\dagger} M\right)\left\{I-\left[N\left(I-M^{\dagger} M\right)\right]^{\dagger}\left[N\left(I-M^{\dagger} M\right)\right]\right\}\right.$. Then, for some $z \in \mathbb{C}^{m}$, one gets

$$
v=\left(I-M^{\dagger} M\right)\left\{I-\left[N\left(I-M^{\dagger} M\right)\right]^{\dagger}\left[N\left(I-M^{\dagger} M\right)\right]\right\} z .
$$

Hence, and using that $M=M M^{\dagger} M$, it follows that

$$
M v=\left(M-M M^{\dagger} M\right)\left\{I-\left[N\left(I-M^{\dagger} M\right)\right]^{\dagger}\left[N\left(I-M^{\dagger} M\right)\right]\right\} z=0
$$

and

$$
N v=\left\{N\left(I-M^{\dagger} M\right)-\left[N\left(I-M^{\dagger} M\right)\right]\left[N\left(I-M^{\dagger} M\right)\right]^{\dagger}\left[N\left(I-M^{\dagger} M\right)\right]\right\} z=0 .
$$

Thus $v \in \operatorname{ker} M \cap \operatorname{ker} N$, and the result is established.

The set of all the real numbers will be denoted by $\mathbb{R}$, and the set of all non-negative integers will be denoted by $\mathbb{N}$. If $A$ is a matrix in $\mathbb{C}^{m \times m}$, we $\operatorname{denote} \beta(A)=\min \{\operatorname{Re}(w)$; $w \in \sigma(A)\}$, and if $\beta(A)>0$ and $t \geqslant 0$, from (1.8) one gets

$$
\left\|\mathrm{e}^{-t A}\right\| \leqslant \mathrm{e}^{-t \beta(A)} \sum_{k=0}^{m-1} \frac{\|\sqrt{m} A\|^{k} t^{k}}{k !}, \quad t \geqslant 0 .
$$

\section{Vector eigenvalue differential systems}

Vector Sturm-Liouville differential systems of the form

$$
\begin{gathered}
-\left(P(x) y^{\prime}\right)^{\prime}+Q(x) y=\lambda W(x) y, \quad a \leqslant x \leqslant b, \\
A_{1}^{*} y(a)+A_{2}^{*} P(a) y^{\prime}(a)=0, \\
B_{1}^{*} y(b)+B_{2}^{*} P(b) y^{\prime}(b)=0,
\end{gathered}
$$

where $P, Q$ and $W$ are symmetric $m \times m$ matrix functions of $x$ with $P$ and $W$ positive definite for all $x \in[a, b], y$ is an $m$-vector function of $x, \lambda$ is a scalar parameter, and $A_{1}, A_{2}, B_{1}$ and $B_{2}$ are matrices in $\mathbb{C}^{m \times m}$, such that $A_{1}^{*} A_{2}=A_{2}^{*} A_{1}, B_{1}^{*} B_{2}=B_{2}^{*} B_{1}$, and $\left(A_{1}, A_{2}\right),\left(B_{1}, B_{2}\right)$ are full-rank $\mathbb{C}^{m \times 2 m}$ matrices which have been treated in $[\mathbf{3}, \mathbf{4}, \mathbf{1 2}, \mathbf{1 8}]$. In this section, we consider vector eigenvalue differential problems of the type (1.7). Suppose that

$$
A_{1}=I
$$


Under this hypothesis, the general solution of the vector equation $X^{\prime \prime}+\lambda^{2} X=0$ is given by

$$
X_{\lambda}(x)= \begin{cases}\sin (\lambda x) D_{\lambda}+\cos (\lambda x) E_{\lambda}, & D_{\lambda}, E_{\lambda} \in \mathbb{C}^{m}, \quad \lambda>0 \\ D_{0}+x E_{0}, & D_{0}, E_{0} \in \mathbb{C}^{m}, \quad \lambda=0 .\end{cases}
$$

Condition $X(0)+B_{1} X^{\prime}(0)=0$ implies $D_{\lambda}=-\lambda B_{1} E_{\lambda}$, if $\lambda>0$ and $D_{0}=-B_{1} E_{0}$. Hence, (2.2) takes the form

$$
X_{\lambda}(x)= \begin{cases}\left(\cos (\lambda x)-\lambda B_{1} \sin (\lambda x)\right) E_{\lambda}, & E_{\lambda} \in \mathbb{C}^{m}, \quad \lambda>0, \\ \left(I x-B_{1}\right) E_{0}, & E_{0} \in \mathbb{C}^{m}, \quad \lambda=0 .\end{cases}
$$

By imposing the remaining boundary-value conditions $A^{j} X(0)+B_{1} A^{j} X^{\prime}(0)=0$ for $1 \leqslant j \leqslant p-1$ and $A_{2} A^{j} X(1)+B_{2} A^{j} X^{\prime}(1)=0,0 \leqslant j \leqslant p-1$, one gets the following conditions on the vector $E_{\lambda}$, for $\lambda>0$ :

$$
\begin{array}{r}
\left(A^{j} B_{1}-B_{1} A^{j}\right) E_{\lambda}=0, \quad 1 \leqslant j \leqslant p-1, \quad \lambda>0 \\
{\left[-\lambda\left(\cos (\lambda) A_{2}-\lambda \sin (\lambda) B_{2}\right) A^{j} B_{1}+\left(\sin (\lambda) A_{2}+\lambda \cos (\lambda) B_{2}\right) A^{j}\right] E_{\lambda}=0} \\
\text { for } 0 \leqslant j \leqslant p-1, \quad \lambda>0 .
\end{array}
$$

Taking into account (2.4), conditions (2.5) can be written in the form

$$
\begin{aligned}
& {\left[\sin (\lambda)\left(A_{2}+\lambda^{2} B_{2} B_{1}\right)+\lambda \cos (\lambda)\left(B_{2}-A_{2} B_{1}\right)\right] A^{j} E_{\lambda}=0,} \\
& \text { for } 0 \leqslant j \leqslant p-1, \quad \lambda>0 .
\end{aligned}
$$

Since we seek non-zero vectors $E_{\lambda}$, by (2.6) one gets that

$$
L(\lambda)=\left(A_{2}+\lambda^{2} B_{2} B_{1}\right) \sin (\lambda)+\left(B_{2}-A_{2} B_{1}\right) \lambda \cos (\lambda) \text { is singular, } \quad \lambda>0 .
$$

Assume that the block matrix

$$
\left[\begin{array}{ll}
I & B_{1} \\
A_{2} & B_{2}
\end{array}\right]
$$

is invertible. By (2.8) and the properties of the Schur complement of a matrix [5, p. 93], one gets that $B_{2}-A_{2} B_{1}$ is invertible, and condition (2.7) implies $\sin (\lambda) \neq 0$. Hence, condition (2.7) is equivalent to

$$
A_{2}+\lambda^{2} B_{2} B_{1}+\lambda \cot (\lambda)\left(B_{2}-A_{2} B_{1}\right) \text { singular, } \quad \lambda>0,
$$

or

$$
\left(B_{2}-A_{2} B_{1}\right)^{-1} A_{2}+\lambda^{2}\left(B_{2}-A_{2} B_{1}\right)^{-1} B_{2} B_{1}+\lambda I \cot (\lambda) I \text { singular, } \quad \lambda>0 .
$$

Hence,

$$
\lambda \cot (\lambda) \in \sigma\left(\left(A_{2} B_{1}-B_{2}\right)^{-1} A_{2}+\lambda^{2}\left(A_{2} B_{1}-B_{2}\right)^{-1} B_{2} B_{1}\right), \quad \lambda>0 .
$$


Let us introduce the matrices

$$
\hat{A}_{2}=\left(A_{2} B_{1}-B_{2}\right)^{-1} A_{2}, \quad \hat{B}_{2}=\left(A_{2} B_{1}-B_{2}\right)^{-1} B_{2},
$$

and note that

$$
\hat{B}_{2}=\hat{A}_{2} B_{1}-I
$$

Hence condition (2.9) can be written in the form

$$
\lambda \cot (\lambda) \in \sigma\left(\hat{A}_{2}+\lambda^{2}\left(\hat{A}_{2} B_{1}^{2}-B_{1}\right)\right), \quad \lambda>0 .
$$

Assume that matrices $\hat{A}_{2}$ and $B_{1}$ have real eigenvalues $\alpha \in \sigma\left(\hat{A}_{2}\right)$ and $\beta \in \sigma\left(B_{1}\right)$ and a common eigenvector $v \in \mathbb{C}^{m}$ associated to them:

$$
\left(B_{1}-\beta I\right) v=\left(\hat{A}_{2}-\alpha I\right) v=0, \quad v \in \mathbb{C}^{m}, \quad v \neq 0, \quad(\alpha, \beta) \in \mathbb{R}^{2} .
$$

Then

$$
\left[\hat{A}_{2}+\lambda^{2}\left(\hat{A}_{2} B_{1}^{2}-B_{1}\right)\right] v=\left[\alpha+\lambda^{2}\left(\alpha \beta^{2}-\beta\right)\right] v
$$

and, for $\lambda>0$, one gets

$$
\left.\begin{array}{l}
\alpha+\lambda^{2}\left(\alpha \beta^{2}-\beta\right) \text { is a real eigenvalue of } \hat{A}_{2}+\lambda^{2}\left(\hat{A}_{2} B_{1}^{2}-B_{1}\right) \\
\text { and } v \text { is an eigenvector associated with } \alpha+\lambda^{2}\left(\alpha \beta^{2}-\beta\right),
\end{array}\right\}
$$

and

$$
\lambda \cot (\lambda)=\alpha+\lambda^{2}\left(\alpha \beta^{2}-\beta\right), \quad \lambda>0,
$$

has a sequence of positive roots. Note that by (2.4) and (2.7), eigenfunctions $X_{\lambda}(x)$ are given by (see (2.3))

$$
X_{\lambda}(x)=\left\{\cos (\lambda x)-\lambda B_{1} \sin (\lambda x)\right\} E_{\lambda}, \quad E_{\lambda} \in \mathbb{C}^{m}, \quad \lambda>0
$$

where vectors $E_{\lambda}$ satisfy

$$
H_{\lambda} E_{\lambda}=0, \quad \lambda>0
$$

where $H_{\lambda}$ is the matrix in $\mathbb{C}^{(2 p-1) m \times m}$ defined by

$$
H_{\lambda}=\left[\begin{array}{c}
B_{1} A-A B_{1} \\
B_{1} A^{2}-A^{2} B_{1} \\
\vdots \\
B_{1} A^{p-1}-A^{p-1} B_{1} \\
\hat{A}_{2}+\lambda^{2}\left(\hat{A}_{2} B_{1}^{2}-B_{1}\right)-\left(\alpha+\lambda^{2}\left(\alpha \beta^{2}-\beta\right)\right) I \\
{\left[\hat{A}_{2}+\lambda^{2}\left(\hat{A}_{2} B_{1}^{2}-B_{1}\right)-\left(\alpha+\lambda^{2}\left(\alpha \beta^{2}-\beta\right)\right) I\right] A} \\
\vdots \\
{\left[\hat{A}_{2}+\lambda^{2}\left(\hat{A}_{2} B_{1}^{2}-B_{1}\right)-\left(\alpha+\lambda^{2}\left(\alpha \beta^{2}-\beta\right)\right) I\right] A^{p-1}}
\end{array}\right] .
$$


If for $\lambda=0$, by imposing to $X_{0}(x)=\left(I x-B_{1}\right) E_{0}$ given by (2.3), the boundary-value conditions $A^{j} X(0)+B_{1} A^{j} X^{\prime}(0)=0$ for $1 \leqslant j \leqslant p-1$ and $A_{2} A^{j} X(1)+B_{2} A^{j} X^{\prime}(1)=0$, $0 \leqslant j \leqslant p-1$, it follows that $E_{0} \in \mathbb{C}^{m}$ must verify

$$
\begin{aligned}
\left(B_{1} A^{j}-A^{j} B_{1}\right) E_{0}=0, & 1 \leqslant j \leqslant p-1, \\
A_{2} A^{j}\left(I-B_{1}\right) E_{0}+B_{2} A^{j} E_{0}=0, & 0 \leqslant j \leqslant p-1 .
\end{aligned}
$$

Note that condition (2.19) is also verified for $j=0$. Substituting condition (2.19) into (2.20) one gets

$$
\begin{gathered}
A_{2} A^{j} E_{0}-A_{2} B_{1} A^{j} E_{0}+B_{2} A^{j} E_{0}=0, \\
\left(A_{2}-A_{2} B_{1}+B_{2}\right) A^{j} E_{0}=0, \quad 0 \leqslant j \leqslant p-1 .
\end{gathered}
$$

By the definition of $\hat{A}_{2}$ given by (2.10), it follows that $A_{2}=\left(A_{2} B_{1}-B_{2}\right) \hat{A}_{2}$, and, thus, condition (2.21) can be written in the form

$$
\left(A_{2} B_{1}-B_{2}\right)\left(\hat{A}_{2}-I\right) A^{j} E_{0}=0, \quad 0 \leqslant j \leqslant p-1 .
$$

Since $A_{2} B_{1}-B_{2}$ is invertible, condition (2.22) is equivalent to

$$
\left(\hat{A}_{2}-I\right) A^{j} E_{0}=0, \quad 0 \leqslant j \leqslant p-1 .
$$

Thus, conditions (2.19) and (2.20) are equivalent to the condition

$$
H_{0} E_{0}=0
$$

where

$$
H_{0}=\left[\begin{array}{c}
B_{1} A-A B_{1} \\
B_{1} A^{2}-A^{2} B_{1} \\
\vdots \\
B_{1} A^{p-1}-A^{p-1} B_{1} \\
\hat{A}_{2}-I \\
\left(\hat{A}_{2}-I\right) A \\
\vdots \\
\left(\hat{A}_{2}-I\right) A^{p-1}
\end{array}\right]
$$

Note that taking $\lambda=0$ and $\alpha=1$ in (2.18), one gets $H_{0}$ defined by (2.24). By (2.17) and (2.23), the existence of eigenfunctions associated with $\lambda \geqslant 0$ is granted if the matrix $H_{\lambda}$, defined by (2.18) for $\lambda>0$ and by (2.24) for $\lambda=0$, satisfies

$$
\operatorname{rank} H_{\lambda}<m, \quad \lambda \geqslant 0 .
$$

Furthermore, under condition (2.25) and Theorem 2.3.2 in [26, p. 24], if equation $H_{\lambda} E_{\lambda}=$ 0 is compatible, its solution set is given by

$$
E_{\lambda}=\left(I-H_{\lambda}^{\dagger} H_{\lambda}\right) S_{\lambda}, \quad S_{\lambda} \in \mathbb{C}^{m}
$$


Assume that apart from condition (2.13), vector $v$ satisfies

$$
\left\{A^{j} v ; 1 \leqslant j \leqslant p-1\right\} \subset \operatorname{Ker}\left(\hat{A}_{2}-\alpha I\right)
$$

then $v$ satisfies $H_{\lambda} v=0$ for all the positive solutions $\lambda$ of equation (2.15). Note that condition (2.26) is granted if apart from (2.13), we assume that

$$
\operatorname{Ker}\left(B_{1}-\beta I\right) \cap \operatorname{Ker}\left(\hat{A}_{2}-\alpha I\right) \text { is an invariant subspace of } A \text {. }
$$

Summarizing, the following result has been established.

Theorem 2.1. Let $A \in \mathbb{C}^{m \times m}, p$ an integer, $p \geqslant 1$, and suppose that the matrix

$$
\left[\begin{array}{ll}
I & B_{1} \\
A_{2} & B_{2}
\end{array}\right]
$$

is invertible in $\mathbb{C}^{2 m \times 2 m}$. Let $\hat{A}_{2}, \hat{B}_{2}$ be defined by (2.10) and assume condition (2.13) for some vector $v \in \mathbb{C}^{m}$. Let $H_{\lambda}$ be defined by (2.18) for $\lambda>0$ and by (2.24) if $\lambda=0$.

(i) A positive solution, $\lambda$, of equation (2.15) is an eigenvalue of problem (1.7) if rank $H_{\lambda}<m$, and $\lambda_{0}=0$ is an eigenvalue if rank $H_{0}<m$.

(ii) If apart from condition (2.13) the vector $v$ satisfies (2.26), then problem (1.7) admits a countable set $\mathcal{F}(\alpha, \beta)=\left\{\lambda_{n} ; n \in \mathbb{N}\right\}$ of real eigenvalues with $\lim _{n \rightarrow \infty} \lambda_{n}=+\infty$.

(iii) If $\lambda_{n} \geqslant 0$ is an eigenvalue of problem (1.7), then eigenfunctions associated to $\lambda_{n}$ are given by

$$
X_{\lambda_{n}}(x)= \begin{cases}\left\{\cos \left(\lambda_{n} x\right)-\lambda_{n} B_{1} \sin \left(\lambda_{n} x\right)\right\} E_{\lambda_{n}}, & \lambda_{n}>0 \\ \left(I x-B_{1}\right) E_{0}, & \lambda_{0}=0\end{cases}
$$

where $E_{\lambda_{n}}=\left(I-H_{\lambda_{n}}^{\dagger} H_{\lambda_{n}}\right) S_{\lambda_{n}}$, where $S_{\lambda_{n}}$ is an arbitrary vector in $\mathbb{C}^{m}$

Remark 2.2. With respect to the localization of the eigenvalues of problem (1.7), it is easy to show that the sequence $\left\{\lambda_{k}\right\}_{k \geqslant 1}$ of non-negative roots of equation (2.15) verifies the following cases.

Case 1. $\beta(1-a)>0$. If

$$
\begin{aligned}
& \alpha>1, \quad k \pi<\lambda_{k}<\frac{1}{2}(2 k+1) \pi, \quad k \geqslant 1, \\
& 0 \leqslant \alpha<1, \quad(k-1) \pi<\lambda_{k}<\frac{1}{2}(2 k-1) \pi, \quad k \geqslant 1 \text {, } \\
& \alpha<0, \quad(k-1) \pi<\lambda_{k}<k \pi, \quad k \geqslant 1 \text {. }
\end{aligned}
$$

Thus, in all the subcases, one gets

$$
(k-1) \pi<\lambda_{k}<k+\frac{1}{2} \pi, \quad k \geqslant 1,
$$


Case 2. $\beta(1-\alpha)=0$. If

$$
\begin{array}{rrrr}
\alpha>1, & k \pi<\lambda_{k}<\frac{1}{2}(2 k+1) \pi, & k \geqslant 1, \\
\alpha=1, & \lambda_{0}=0 \text { and } k \pi<\lambda_{k}<\frac{1}{2}(2 k+1) \pi, & k \geqslant 1, \\
0<\alpha<1, & (k-1) \pi<\lambda_{k}<\frac{1}{2}(2 k-1) \pi, & k \geqslant 1, \\
\alpha=0, & \lambda_{k}=\frac{1}{2}(2 k-1) \pi, & k \geqslant 1, \\
\alpha<0, & \frac{1}{2}(2 k-1) \pi<\lambda_{k}<k \pi, & k \geqslant 1 .
\end{array}
$$

So in all the subcases for $k \geqslant 1$ one gets $(2.27)$.

Case 3. $\beta(1-\alpha)<0$. If

$$
\begin{aligned}
& \alpha>1, \quad(k-1) \pi<\lambda_{k}<(k+1) \pi, \quad k \geqslant 1, \\
& 0<\alpha<1, \quad(k-1) \pi<\lambda_{k}<k \pi, \quad k \geqslant 1, \\
& \alpha \leqslant 0, \quad \frac{1}{2}(2 k-1) \pi<\lambda_{k}<k \pi, \quad k \geqslant 1 .
\end{aligned}
$$

Thus, in all the cases the positive solutions $\lambda_{k}$ of (2.15) verify (2.27).

Remark 2.3. The study of the problem with $B_{1}=I$,

$$
\left.\begin{array}{c}
X^{\prime \prime}(x)+\lambda^{2} X(x)=0, \quad 0<x<1, \\
A_{1} A^{j} X(0)+A^{j} X^{\prime}(0)=0, \\
A_{2} A^{j} X(1)+B_{2} A^{j} X^{\prime}(1)=0, \\
0 \leqslant j \leqslant p-1, \quad p \geqslant 1,
\end{array}\right\}
$$

is analogous to problem (1.7). It is easy to check that the problems

$$
\left.\begin{array}{c}
X^{\prime \prime}(x)+\lambda^{2} X(x)=0, \quad 0<x<1, \\
A_{1} A^{j} X(0)+B_{1} A^{j} X^{\prime}(0)=0, \\
A_{2} A^{j} X(1)+B_{2} A^{j} X^{\prime}(1)=0, \\
0 \leqslant j \leqslant p-1, \quad p \geqslant 1,
\end{array}\right\}
$$

where $A_{2}=I$ or $B_{2}=I$, can be reduced to the previous cases considering the change of variables defined by

$$
y=y(x)=1-x, \quad 0 \leqslant x \leqslant 1
$$

Thus, the approach developed is applicable to any problem of the type

$$
\left.\begin{array}{c}
X^{\prime \prime}(x)+\lambda^{2} X(x)=0, \quad 0<x<1, \\
A_{1} A^{j} X(0)+B_{1} A^{j} X^{\prime}(0)=0, \\
A_{2} A^{j} X(1)+B_{2} A^{j} X^{\prime}(1)=0, \\
0 \leqslant j \leqslant p-1, \quad p \geqslant 1,
\end{array}\right\}
$$


where some of the block entries are the identity matrix. Finally, it is important to point out that the hypothesis $A_{i}=I$ for $i=1$ or $i=2$, or $B_{i}=I$ for $i=1$ or $i=2$, does not involve a lack of generality. In fact, if in problem (2.31) one verifies that some $A_{i}$ (respectively, $B_{i}$ ) is invertible, premultiplying the corresponding boundary condition of (2.31) by $A_{i}^{-1}$ (respectively, $B_{i}^{-1}$ ), one achieves a previously considered problem.

\section{Construction of an exact series solution}

Let us seek solutions of the boundary-value problems (1.1)-(1.3) under hypotheses (2.1) and (2.8). A separation-of-variables technique suggests

$$
v_{\lambda}(x, t)=T_{\lambda}(t) X_{\lambda}(x), \quad T_{\lambda}(t) \in \mathbb{C}^{m \times m}, \quad X_{\lambda}(x) \in \mathbb{C}^{m}, \quad \lambda \geqslant 0
$$

where

$$
\begin{aligned}
T_{\lambda}^{\prime}(t)+\lambda^{2} A T_{\lambda}(t) & =0, \quad t \geqslant 0, \quad \lambda \geqslant 0, \\
X_{\lambda}^{\prime \prime}(x)+\lambda^{2} X_{\lambda}(x) & =0, \quad 0<x<1, \quad \lambda \geqslant 0, \\
X_{\lambda}(0)+B_{1} X_{\lambda}^{\prime}(0) & =0 \\
A_{2} X_{\lambda}(1)+B_{2} X_{\lambda}^{\prime}(1) & =0 .
\end{aligned}
$$

The solution of (3.2) satisfying $T_{\lambda}(0)=I$ is $T_{\lambda}(t)=\exp \left(-\lambda^{2} A t\right)$, but, although $v_{\lambda}(x, t)$ defined by (3.1) satisfies (1.1)

$$
\begin{aligned}
\frac{\partial}{\partial t}\left(v_{\lambda}(x, t)\right)-A \frac{\partial^{2}}{\partial x^{2}}\left(v_{\lambda}(x, t)\right) & =T_{\lambda}^{\prime}(t) X_{\lambda}(x)-A T_{\lambda}(t) X_{\lambda}^{\prime \prime}(x) \\
& =-\lambda^{2} A T_{\lambda}(t) X_{\lambda}(x)+A T_{\lambda}(t) \lambda^{2} X_{\lambda}(x)=0
\end{aligned}
$$

condition (1.2) is not granted because

$$
\begin{aligned}
v_{\lambda}(0, t)+B_{1} \frac{\partial}{\partial x}\left(v_{\lambda}(0, t)\right) & =T_{\lambda}(t) X_{\lambda}(0)+B_{1} T_{\lambda}(t) X_{\lambda}^{\prime}(0) \\
& =\exp \left(-\lambda^{2} A t\right) X_{\lambda}(0)+B_{1} \exp \left(-\lambda^{2} A t\right) X_{\lambda}^{\prime}(0)
\end{aligned}
$$

and the last equation does not vanish because matrix $B_{1}$ does not commute with $A$. However, if $X_{\lambda}$ satisfies (1.7) instead of (3.3), where $p$ is the degree of the minimal polynomial of $A$, then $T_{\lambda}(t)=\exp \left(-\lambda^{2} A t\right)$ can be expressed as a matrix polynomial of $A[9$, p. 557],

$$
T_{\lambda}(t)=\exp \left(-\lambda^{2} A t\right)=b_{0}(t, \lambda) I+b_{1}(t, \lambda) A+\cdots+b_{p-1}(t, \lambda) A^{p-1},
$$

where $b_{j}(t, \lambda), 0 \leqslant j \leqslant p-1$ are scalars. Under the boundary-value conditions of (1.7) it follows that

$$
v_{\lambda}(0, t)+B_{1} \frac{\partial}{\partial x}\left(v_{\lambda}(0, t)\right)=\sum_{j=0}^{p-1} b_{j}(t, \lambda)\left\{A^{j} X_{\lambda}(0)+B_{1} A^{j} X_{\lambda}^{\prime}(0)\right\}=0, \quad t \geqslant 0
$$


and

$$
A_{2} v_{\lambda}(1, t)+B_{2} \frac{\partial}{\partial x}\left(v_{\lambda}(1, t)\right)=\sum_{j=0}^{p-1} b_{j}(t, \lambda)\left\{A_{2} A^{j} X_{\lambda}(1)+B_{1} A^{j} X_{\lambda}^{\prime}(1)\right\}=0, \quad t \geqslant 0 .
$$

Assume the notation and hypotheses of Theorem 2.1 and let $\left\{\lambda_{n}\right\}_{n=1}^{\infty}$ be the sequence of positive eigenvalues of problem (1.7). The candidate series solution of problems (1.1)(1.4) is given by

$$
U(x, t)= \begin{cases}X_{0}(x) E_{0}+\sum_{n \geqslant 1} \exp \left(-\lambda_{n}^{2} A t\right) X_{\lambda_{n}}(x), & 0 \in \mathcal{F}(\alpha, \beta), \\ \sum_{n \geqslant 1} \exp \left(-\lambda_{n}^{2} A t\right) X_{\lambda_{n}}(x), & 0 \notin \mathcal{F}(\alpha, \beta),\end{cases}
$$

where $X_{\lambda_{n}}$ is defined by Theorem 2.1, for appropriate vectors $E_{\lambda_{n}}$ to be determined. Consider the case where $0 \notin \mathcal{F}(\alpha, \beta)$. Associated to problem (1.7) we introduce the scalar Sturm-Liouville problem

$$
\left.\begin{array}{c}
X^{\prime \prime}(x)+\lambda^{2} X(x)=0, \quad 0<x<1 \\
X(0)+\beta X^{\prime}(0)=0 \\
\alpha X(1)+(\alpha \beta-1) X^{\prime}(1)=0 .
\end{array}\right\}
$$

For the sake of well-posedness, assume that function $f(x)$ appearing in (1.4) satisfies the property

$$
\left.\begin{array}{l}
f(x) \text { is twice continuously differentiable in }[0,1] \\
\text { and } f(0)+\beta f^{\prime}(0)=0, \alpha f(1)+(\alpha \beta-1) f^{\prime}(1)=0 .
\end{array}\right\}
$$

By the convergence theorem in series of Sturm-Liouville functions (see [14, ch. 11], $\left[10\right.$, p. 90] and [7]), each component $f_{i}(x)$ of $f$, for $1 \leqslant i \leqslant m$ admits a series representation, absolute and uniformly convergent in $[0,1]$, of the form

$$
f_{i}(x)=\sum_{n \geqslant 1}\left\{\sin \left(\lambda_{n} x\right)+\lambda_{n} \beta \cos \left(\lambda_{n} x\right)\right\} e_{\lambda_{n}}(i), \quad 0 \leqslant x \leqslant 1,
$$

where

$$
e_{\lambda_{n}}(i)=\frac{\int_{0}^{1} f_{i}(x)\left\{\sin \left(\lambda_{n} x\right)+\beta \lambda_{n} \cos \left(\lambda_{n} x\right)\right\} \mathrm{d} x}{\int_{0}^{1}\left\{\sin \left(\lambda_{n} x\right)+\beta \lambda_{n} \cos \left(\lambda_{n} x\right)\right\}^{2} \mathrm{~d} x}, \quad n \geqslant 1, \quad 1 \leqslant i \leqslant m
$$

Note that if we define vectors $E_{\lambda_{n}} \in \mathbb{C}^{m}$ by

$$
E_{\lambda_{n}}=\left[\begin{array}{c}
e_{\lambda_{n}}(1) \\
e_{\lambda_{n}}(2) \\
\vdots \\
e_{\lambda_{n}}(m)
\end{array}\right]
$$


then $U(x, t)$, defined by

$$
U(x, t)=\sum_{n \geqslant 1} \exp \left(-\lambda_{n}^{2} A t\right)\left\{\sin \left(\lambda_{n} x\right)-\beta \lambda_{n} \cos \left(\lambda_{n} x\right)\right\} E_{\lambda_{n}}
$$

satisfies $U(x, 0)=f(x), 0 \leqslant x \leqslant 1$.

For the case where $0 \in \mathcal{F}(\alpha, \beta)$ and $\lambda_{0}=0$ is an eigenvalue, we consider the scalar Sturm-Liouville problem (3.7) with $\alpha=1$ :

$$
\left.\begin{array}{c}
X^{\prime \prime}(x)+\lambda^{2} X(x)=0, \quad 0<x<1, \\
X(0)+\beta X^{\prime}(0)=0 \\
\alpha X(1)+(\beta-1) X^{\prime}(1)=0 .
\end{array}\right\}
$$

If function $f(x)$ appearing in (1.4) satisfies condition (3.8) with $\alpha=1$, and if apart from $e_{\lambda_{n}}(i)$, defined by $(3.10)$, one considers

$$
e_{0}(i)=\frac{\int_{0}^{1} f_{i}(x)(x-\beta) \mathrm{d} x}{\int_{0}^{1}(x-\beta)^{2} \mathrm{~d} x}, \quad 1 \leqslant i \leqslant m, \quad E_{0}=\left[\begin{array}{c}
e_{0}(1) \\
e_{0}(2) \\
\vdots \\
e_{0}(m)
\end{array}\right],
$$

then $U(x, t)$, defined by

$$
U(x, t)=(x-\beta) E_{0}+\sum_{n \geqslant 1} \exp \left(-\lambda_{n}^{2} A t\right)\left\{\sin \left(\lambda_{n} x\right)-\beta \lambda_{n} \cos \left(\lambda_{n} x\right)\right\} E_{\lambda_{n}}
$$

satisfies the initial condition (1.4). Note that in order to satisfy conditions (1.1)-(1.3), vectors $E_{\lambda_{n}}$ must verify the conditions of Theorem 2.1. By definition of vector $E_{\lambda_{n}}$, these conditions are satisfied if

$$
H_{0} f(x)=0, \quad H_{\lambda_{n}} f(x)=0, \quad\left(B_{1}-\beta I\right) f(x)=0, \quad 0 \leqslant x \leqslant 1 .
$$

Note that by definition of $H_{\lambda_{n}}$, condition (3.15) holds if

$$
\left(\hat{A}_{2}-\alpha I\right) A^{j} f(x)=0=\left(B_{1}-\beta I\right) A^{j} f(x), \quad 0 \leqslant x \leqslant 1, \quad 0 \leqslant j \leqslant p-1 .
$$

Conversely, if the conditions in (3.15) hold true, then

$$
\left(B_{1} A^{j}-A^{j} B_{1}\right) f(x)=\left(B_{1}-\beta I\right), \quad 0 \leqslant x \leqslant 1, \quad 0 \leqslant j \leqslant p-1
$$

and

$$
\begin{aligned}
\left\{\hat{A}_{2}+\lambda^{2}\left(\hat{A}_{2} B_{1}^{2}\right.\right. & \left.\left.-B_{1}\right)-\left[\alpha+\lambda^{2}\left(\alpha \beta^{2}-\beta\right)\right] I\right\} A^{j} f(x) \\
& =\left\{\hat{A}_{2}+\lambda^{2}\left(\hat{A}_{2} \beta_{1}^{2}-\beta I\right)-\left[\alpha+\lambda^{2}\left(\alpha \beta^{2}-\beta\right)\right] I\right\} A^{j} f(x) \\
& =\left(1+\lambda^{2} \beta^{2}\right)\left(\hat{A}_{2}-\alpha I\right) A^{j} f(x)=0, \quad 0 \leqslant x \leqslant 1, \quad 0 \leqslant j \leqslant p-1 .
\end{aligned}
$$


Thus, conditions (3.15) and (3.16) are equivalent, and it is clear that (3.16) is equivalent to the condition

$$
\left.\begin{array}{l}
\left(\hat{A}_{2}-\alpha I\right) f(x)=0=\left(B_{1}-\beta I\right) f(x), 0 \leqslant x \leqslant 1, \text { and } \\
\operatorname{ker}\left(\hat{A}_{2}-\alpha I\right) \cap \operatorname{ker}\left(B_{1}-\beta I\right) \text { is an invariant subspace of } A .
\end{array}\right\}
$$

By Lemma 1.1, taking $M=\hat{A}_{2}-\alpha I, N=B_{1}-\beta I$, condition (3.17) can be written in the compact form

$$
f(x) \in \operatorname{Im} H(\alpha, \beta), \quad 0 \leqslant x \leqslant 1, \quad \text { and } \quad\left[I-H(\alpha, \beta)(H(\alpha, \beta))^{\dagger}\right] A H(\alpha, \beta)=0,
$$

where

$$
\left.\begin{array}{c}
H(\alpha, \beta)=\left(I-M_{\alpha}^{\dagger} M_{\alpha}\right)\left\{I-\left[N_{\beta}\left(I-M_{\alpha}^{\dagger} M_{\alpha}\right)\right]^{\dagger}\left[N_{\beta}\left(I-M_{\alpha}^{\dagger} M_{\alpha}\right)\right]\right\}, \\
M_{\alpha}=\hat{A}_{2}-\alpha I, \quad N_{\beta}=B_{1}-\beta I .
\end{array}\right\}
$$

Note that condition (3.18) means that $f(x)$ lies in $\operatorname{Im} H(\alpha, \beta)$ and that $\operatorname{Im} H(\alpha, \beta)$ is an invariant subspace of the matrix $A$. With respect to the convergence of the series (3.11) or (3.14) -as well as their partial differentiability with respect to the variable $t$ once, and $x$ twice, for $0<x<1, t>0$-note that if $t_{0}>0$ and $D\left(t_{0}\right)=\{(x, t) ; 0 \leqslant x \leqslant 1$, $\left.t \geqslant t_{0}>0\right\}$ by inequality (1.8) and condition (1.5), the series appearing by twice termwise partial differentiation with respect to $x$ and once with respect to $t$, in (3.11), takes the form

$$
\sum_{n \geqslant 1} \lambda_{n}^{2} \exp \left(-\lambda_{n}^{2} A t\right) X_{\lambda_{n}}(x), \quad \sum_{n \geqslant 1}\left(-\lambda_{n}^{2}\right) A \exp \left(-\lambda_{n}^{2} A t\right) X_{\lambda_{1 \prime}}(x)
$$

and is uniformly convergent in $D\left(t_{0}\right)$. By the differentiation theorem of functional series $[2$, p. 403], the series (3.11) or (3.14) define rigorous solutions of problems (1.1)-(1.4), and the following result has been established.

Theorem 3.1. Let $A$ be a positive stable matrix in $\mathbb{C}^{m \times m}$, assume that

$$
\left[\begin{array}{cc}
I & B_{1} \\
A_{2} & B_{2}
\end{array}\right]
$$

is invertible and that there exist real numbers $\alpha$ and $\beta$ satisfying (2.13). If $\hat{A}_{2}$ and $\hat{B}_{2}$ are defined by (2.10), $H(\alpha, \beta)$ by (3.19), and $f(x)$ is twice continuously differentiable in $[0,1]$, satisfying (3.8) and (3.18), then problems (1.1)-(1.4) admit a well-posed solution given by (3.11) or (3.14), where vectors $E_{\lambda_{n}}$ are defined by (3.10) for $n \geqslant 1$ and by (3.13) for $n=0$.

Remark 3.2. Condition (3.8) together with (3.18) are equivalent to

$$
\left.\begin{array}{rl}
f(0)+B_{1} f^{\prime}(0) & =0 \\
A_{2} f(1)+B_{2} f^{\prime}(1) & =0
\end{array}\right\}
$$

and (3.18). In fact, premultiplying the second condition of $\left(3.8^{\prime}\right)$ by $\left(A_{2} B_{1}-B_{2}\right)^{-1}$ and taking into account (2.11), one gets (3.8). 
Now we are interested in the construction of an exact series solution of problems (1.1)(1.4) for more general functions $f(x)$ than those considered in Theorem 3.1. Assume that

$$
\left.\begin{array}{l}
\Lambda=\{\alpha(1), \cdots, \alpha(k)\} \text { are the distinct real eigenvalues of } \hat{A}_{2}, \\
\Omega=\{\beta(1), \cdots, \beta(k)\} \text { are the distinct real eigenvalues of } B_{1},
\end{array}\right\}
$$

and let $H(\alpha(i), \beta(j))$ be the matrix defined by $(3.19)$ for $1 \leqslant i \leqslant k, 1 \leqslant j \leqslant s$. Recall that by Lemma 1.1 , condition $\operatorname{ker}\left(\hat{A}_{2}-\alpha(i) I\right) \cap \operatorname{ker}\left(B_{1}-\beta(j) I\right) \neq 0$ is equivalent to the condition $H(\alpha(i), \beta(j)) \neq 0$. Consider the subset of $\Lambda \times \Omega$ defined by

$$
\mathcal{S}=\left\{\left(\alpha\left(i_{l}\right), \beta\left(j_{l}\right)\right) \in \Lambda \times \Omega ; H\left(\alpha\left(i_{l}\right), \beta\left(j_{l}\right)\right) \neq 0,1 \leqslant l \leqslant q\right\},
$$

and the block matrix in $\mathbb{C}^{m \times m q}$ defined by

$$
\mathcal{H}=\left[H\left(\alpha\left(i_{1}\right), \beta\left(j_{1}\right)\right), H\left(\alpha\left(i_{2}\right), \beta\left(j_{2}\right)\right), \cdots, H\left(\alpha\left(i_{q}\right), \beta\left(j_{q}\right)\right)\right] .
$$

Assume that $f(x)$ is twice continuously differentiable in $[0,1]$ such that

$$
\left.\begin{array}{c}
\left(I-H H^{\dagger}\right) f(x)=0, \quad 0 \leqslant x \leqslant 1, \\
H^{\dagger}\left(f(0)+\beta\left(j_{l}\right) f^{\prime}(0)\right)=0, \\
{\left[0 \cdots 0 H\left(\alpha\left(i_{l}\right), \beta\left(j_{l}\right)\right) 0 \cdots 0\right] H^{\dagger}\left[\alpha\left(i_{l}\right) f(1)+\left(\alpha\left(i_{l}\right) \beta\left(j_{l}\right)-1\right) f^{\prime}(1)\right]=0,}
\end{array}\right\} \quad 1 \leqslant l \leqslant q .
$$

Since, by Lemma 1.1, one gets

$$
\operatorname{Im} H\left(\alpha\left(i_{l}\right), \beta\left(j_{l}\right)\right)=\operatorname{ker}\left(\hat{A}_{2}-\alpha(i) I\right) \cap \operatorname{ker}\left(B_{1}-\beta(j) I\right),
$$

then $\operatorname{Im} H$ is the direct sum of the subspaces $S_{l}=\operatorname{Im} H\left(\alpha\left(i_{l}\right), \beta\left(j_{l}\right)\right), 1 \leqslant l \leqslant q$, and the projection $g_{l}(x)$ of the $f(x)$ on the subspace $S_{l}$ is given by

$$
g_{l}(x)=\left[0 \cdots 0 H\left(\alpha\left(i_{l}\right), \beta\left(j_{l}\right)\right) 0 \cdots 0\right] H^{\dagger} f(x), \quad 1 \leqslant l \leqslant q, \quad 0 \leqslant x \leqslant 1,
$$

because

$$
g_{l}(x) \in \operatorname{Im} H\left(\alpha\left(i_{l}\right), \beta\left(j_{l}\right)\right)=S_{l}
$$

and, by (3.23), one gets

$$
\sum_{l=1}^{q} g_{l}(x)=H H^{\dagger} f(x), \quad 0 \leqslant x \leqslant 1 .
$$

By the hypothesis on $f(x)$, it follows that $g_{l}(x)$ is twice continuously differentiable in $[0,1]$, and, by $(3.24)$, one gets

$$
\left.\begin{array}{rl}
g_{l}(0)+\beta\left(j_{l}\right) g_{l}^{\prime}(0) & =0 \\
\alpha\left(i_{l}\right) g_{l}(1)+\left(\alpha\left(i_{l}\right) \beta\left(j_{l}\right)-1\right) g_{l}^{\prime}(1) & =0, \quad 1 \leqslant l \leqslant q,
\end{array}\right\}
$$


If the subspace $\operatorname{Im} H\left(\alpha\left(i_{l}\right), \beta\left(j_{l}\right)\right)$ is invariant by the matrix $A_{\text {, or }}$

$$
I-\left[H\left(\alpha\left(i_{l}\right), \beta\left(j_{l}\right)\right)\left[H\left(\alpha\left(i_{l}\right), \beta\left(j_{l}\right)\right)\right]^{\dagger}\right] A H\left(\alpha\left(i_{l}\right), \beta\left(j_{l}\right)\right)=0, \quad 1 \leqslant l \leqslant q,
$$

by (3.26), (3.27) and (3.29) together with Theorem 3.1, one gets a series $U(x, t, l)$ defined by

$$
U(x, t, l)=\left\{\begin{array}{cc}
\sum_{n \geqslant 1} \mathrm{e}^{\left(-\lambda_{n}^{2}(l) A t\right)}\left\{\sin \lambda_{n}(l) x-\beta\left(j_{l}\right) \lambda_{n}(l) \cos \lambda_{n}(l) x\right\} E_{\lambda_{n}(l)}+\left(x-\beta\left(j_{l}\right)\right) E_{0}(l), & 0 \in \mathcal{F}\left(\alpha\left(i_{l}\right), \beta\left(j_{l}\right)\right), \\
\sum_{n \geqslant 1} \mathrm{e}^{\left(-\lambda_{n}^{2}(l) A t\right)}\left\{\sin \lambda_{n}(l) x-\beta\left(j_{l}\right) \lambda_{n}(l) \cos \lambda_{n}(l) x\right\} E_{\lambda_{n}(l)}, & 0 \notin \mathcal{F}\left(\alpha\left(i_{l}\right), \beta\left(j_{l}\right)\right),
\end{array}\right.
$$

where $\mathcal{F}\left(\alpha\left(i_{l}\right), \beta\left(j_{l}\right)\right), \lambda_{n}(l)$ and $E_{\lambda_{n}(l)}$ are given by Theorem 3.1, is a solution of problems (1.1)-(1.3) together with the initial condition

$$
U(x, 0, l)=g_{l}(x), \quad 0 \leqslant x \leqslant 1 .
$$

By (3.28) and (3.32), one gets that

$$
u(x, t)=\sum_{l=1}^{q} U(x, t, l),
$$

is a solution of problems (1.1)-(1.4). Summarizing, the following result has been established.

Theorem 3.3. Let $A$ be a matrix in $\mathbb{C}^{m \times m}$ satisfying (1.5), and assume hypothesis (1.6), where $A_{1}=I$. Let $\mathcal{S}$ and $\mathcal{H}$ be defined by (3.21) and (3.22), respectively. Let $\hat{A}_{2}$ be defined by (2.10), and $f(x)$ is a twice continuously differentiable function in [0,1] satisfying (3.23) and (3.24). Under hypothesis (3.30), $u(x, t)$-defined by (3.33), where $U(x, t, l)$ is defined by (3.31), $1 \leqslant l \leqslant q$-is a solution of problems (1.1)-(1.4), with $A_{1}=I$.

Remark 3.4. Taking into account Remark 2.3, a solution of problems (1.1)-(1.4) can be constructed in an analogous way under the hypotheses (1.6) and (1.5) and certain conditions on $f(x)$.

The following example illustrates that the hypotheses of Theorem 3.3 are easy to check.

Example 3.5. Consider problems (1.1)-(1.4), where $A_{1}=I$ in $\mathbb{C}^{4 \times 4}$,

$$
A_{2}=\left[\begin{array}{rrrr}
0 & 0 & 0 & 0 \\
-2 & 0 & 2 & 0 \\
1 & 0 & 0 & -1 \\
0 & 0 & 0 & 0
\end{array}\right], \quad B_{1}=\left[\begin{array}{rrrr}
1 & 0 & 0 & -2 \\
0 & 2 & 0 & 0 \\
1 & 0 & 1 & -3 \\
-0 & 0 & 0 & -1
\end{array}\right]
$$




$$
B_{2}=\left[\begin{array}{rrrr}
0 & 0 & 0 & -1 \\
0 & 0 & 1 & -2 \\
1 & -1 & 0 & -1 \\
-1 & 0 & 0 & 0
\end{array}\right], \quad A=\left[\begin{array}{llll}
2 & 0 & 0 & 0 \\
0 & 2 & 0 & 0 \\
0 & 0 & 1 & 1 \\
1 & 0 & 0 & 1
\end{array}\right]
$$

Here, the block matrix

$$
\left[\begin{array}{cc}
I & B_{1} \\
A_{2} & B_{2}
\end{array}\right]
$$

is invertible, with

$$
\begin{gathered}
\hat{A}_{2}=\left(A_{2} B_{1}-B_{2}\right)^{-1} A_{2}=\left[\begin{array}{rrrr}
0 & 0 & 0 & 0 \\
1 & 0 & 0 & -1 \\
-2 & 0 & 2 & 0 \\
0 & 0 & 0 & 0
\end{array}\right], \\
\sigma(A)=\{1,2\}, \quad \sigma\left(B_{1}\right)=\{2,-1,1\} \quad \text { and } \sigma\left(\hat{A}_{2}\right)=\{0,2\} .
\end{gathered}
$$

With the above notation we have

$$
\begin{aligned}
& M_{0}=\hat{A}_{2}, \quad M_{2}=\hat{A}_{2}-2 I=\left[\begin{array}{rrrr}
-2 & 0 & 0 & 0 \\
1 & -2 & 0 & -1 \\
-2 & 0 & 0 & 0 \\
0 & 0 & 0 & -2
\end{array}\right] \\
& N_{2}=B_{1}-2 I=\left[\begin{array}{rrrr}
-1 & 0 & 0 & -2 \\
0 & 0 & 0 & 0 \\
1 & 0 & -1 & 3 \\
0 & 0 & 0 & -3
\end{array}\right], \quad N_{-1}=B_{1}+I=\left[\begin{array}{rrrr}
2 & 0 & 0 & -2 \\
0 & 3 & 0 & 0 \\
1 & 0 & 2 & -3 \\
0 & 0 & 0 & 0
\end{array}\right] \\
& N_{1}=B_{1}-I=\left[\begin{array}{rrrr}
0 & 0 & 0 & -2 \\
0 & 1 & 0 & 0 \\
1 & 0 & 0 & -3 \\
0 & 0 & 0 & -2
\end{array}\right], \quad M_{0}^{\dagger}=\frac{1}{6}\left[\begin{array}{rrrr}
0 & 2 & -1 & 0 \\
0 & 0 & 0 & 0 \\
0 & 2 & 2 & 0 \\
0 & -4 & -1 & 0
\end{array}\right] \\
& M_{2}^{\dagger}=\frac{1}{8}\left[\begin{array}{rrrr}
-2 & 0 & -2 & 0 \\
-1 & -4 & -1 & 2 \\
0 & 0 & 0 & 0 \\
0 & 0 & 0 & -4
\end{array}\right], \quad N_{2}\left(I-M_{0}^{\dagger} M_{0}\right)=\left[\begin{array}{rrrr}
-1 & 0 & -1 & -1 \\
0 & 0 & 0 & 0 \\
-1 & 0 & -1 & -1 \\
-1 & 0 & -1 & -1
\end{array}\right] \\
& N_{2}\left(I-M_{2}^{\dagger} M_{2}\right)=\left[\begin{array}{rrrr}
0 & 0 & 0 & 0 \\
0 & 0 & 0 & 0 \\
0 & 0 & -1 & 0 \\
0 & 0 & 0 & 0
\end{array}\right], \quad N_{-1}\left(I-M_{0}^{\dagger} M_{0}\right)=\left[\begin{array}{llll}
0 & 0 & 0 & 0 \\
0 & 3 & 0 & 0 \\
0 & 0 & 0 & 0 \\
0 & 0 & 0 & 0
\end{array}\right] \text {, } \\
& N_{-1}\left(I-M_{2}^{\dagger} M_{2}\right)=\left[\begin{array}{llll}
0 & 0 & 0 & 0 \\
0 & 0 & 0 & 0 \\
0 & 0 & 2 & 0 \\
0 & 0 & 0 & 0
\end{array}\right], \quad N_{1}\left(I-M_{0}^{\dagger} M_{0}\right)=\frac{2}{3}\left[\begin{array}{rrrr}
-1 & 0 & -1 & -1 \\
0 & \frac{3}{2} & 0 & 0 \\
-1 & 0 & -1 & -1 \\
-1 & 0 & -1 & -1
\end{array}\right] \text {, }
\end{aligned}
$$




$$
N_{1}\left(I-M_{2}^{\dagger} M_{2}\right)=O \text {. }
$$

Hence,

$$
\begin{gathered}
{\left[N_{2}\left(I-M_{0}^{\dagger} M_{0}\right)\right]^{\dagger}=\frac{1}{9}\left[\begin{array}{rrrr}
-1 & 0 & -1 & -1 \\
0 & 0 & 0 & 0 \\
-1 & 0 & -1 & -1 \\
-1 & 0 & -1 & -1
\end{array}\right], \quad\left[N_{2}\left(I-M_{2}^{\dagger} M_{2}\right)\right]^{\dagger}=\left[\begin{array}{rrrr}
0 & 0 & 0 & 0 \\
0 & 0 & 0 & 0 \\
0 & 0 & -1 & 0 \\
0 & 0 & 0 & 0
\end{array}\right],} \\
{\left[N_{-1}\left(I-M_{0}^{\dagger} M_{0}\right)\right]^{\dagger}=\left[\begin{array}{rrrr}
0 & 0 & 0 & 0 \\
0 & \frac{1}{3} & 0 & 0 \\
0 & 0 & 0 & 0 \\
0 & 0 & 0 & 0
\end{array}\right], \quad\left[N_{-1}\left(I-M_{2}^{\dagger} M_{2}\right)\right]^{\dagger}=\left[\begin{array}{llll}
0 & 0 & 0 & 0 \\
0 & 0 & 0 & 0 \\
0 & 0 & \frac{1}{2} & 0 \\
0 & 0 & 0 & 0
\end{array}\right],} \\
{\left[N_{1}\left(I-M_{0}^{\dagger} M_{0}\right)\right]^{\dagger}=\frac{1}{6}\left[\begin{array}{rrrr}
-1 & 0 & -1 & -1 \\
0 & 1 & 0 & 0 \\
-1 & 0 & -1 & -1 \\
-1 & 0 & -1 & -1
\end{array}\right], \quad\left[N_{1}\left(I-M_{2}^{\dagger} M_{2}\right)\right]^{\dagger}=O .}
\end{gathered}
$$

Matrices $H(\alpha, \beta)$ defined by (3.19) take the values

$$
\begin{gathered}
H(0,2)=\left[\begin{array}{llll}
0 & 0 & 0 & 0 \\
0 & 1 & 0 & 0 \\
0 & 0 & 0 & 0 \\
0 & 0 & 0 & 0
\end{array}\right], \quad H(0,-1)=\frac{1}{3}\left[\begin{array}{llll}
1 & 0 & 1 & 1 \\
0 & 0 & 0 & 0 \\
1 & 0 & 1 & 1 \\
1 & 0 & 1 & 1
\end{array}\right], \\
H(2,1)=\left[\begin{array}{llll}
0 & 0 & 0 & 0 \\
0 & 0 & 0 & 0 \\
0 & 0 & 1 & 0 \\
0 & 0 & 0 & 0
\end{array}\right], \quad H(2,2)=H(2,-1)=H(0,1)=O .
\end{gathered}
$$

Matrix $H$ defined by (3.22) is

$$
H=\left[\begin{array}{llllllllllll}
0 & 0 & 0 & 0 & \frac{1}{3} & 0 & \frac{1}{3} & \frac{1}{3} & 0 & 0 & 0 & 0 \\
0 & 1 & 0 & 0 & 0 & 0 & 0 & 0 & 0 & 0 & 0 & 0 \\
0 & 0 & 0 & 0 & \frac{1}{3} & 0 & \frac{1}{3} & \frac{1}{3} & 0 & 0 & 1 & 0 \\
0 & 0 & 0 & 0 & \frac{1}{3} & 0 & \frac{1}{3} & \frac{1}{3} & 0 & 0 & 0 & 0
\end{array}\right],
$$

and

$$
\begin{gathered}
{\left[\begin{array}{lll}
H(0,2) & 0 & 0
\end{array}\right] H^{\dagger}=\left[\begin{array}{lllr}
0 & 0 & 0 & 0 \\
0 & 1 & 0 & 0 \\
0 & 0 & 0 & 0 \\
0 & 0 & 0 & 0
\end{array}\right],} \\
{\left[\begin{array}{lll}
0 & 0 & H(2,1)
\end{array}\right] H^{\dagger}=\frac{1}{2}\left[\begin{array}{rrrr}
0 & 0 & 0 & 0 \\
0 & 0 & 0 & 0 \\
-1 & 0 & 2 & -1 \\
0 & 0 & 0 & 0
\end{array}\right],}
\end{gathered}
$$




$$
\left[\begin{array}{lll}
0 & H(0,-1) & 0
\end{array}\right] H^{\dagger}=\frac{1}{2}\left[\begin{array}{llll}
1 & 0 & 0 & 1 \\
0 & 0 & 0 & 0 \\
1 & 0 & 0 & 1 \\
1 & 0 & 0 & 1
\end{array}\right], \quad I-H H^{\dagger}=\frac{1}{2}\left[\begin{array}{rrrr}
1 & 0 & 0 & -1 \\
0 & 0 & 0 & 0 \\
0 & 0 & 0 & 0 \\
-1 & 0 & 0 & 0
\end{array}\right] .
$$

If we impose on $f=\left(f_{1}, f_{2}, f_{3}, f_{4}\right)^{\mathrm{T}}$ the condition (3.23), it follows that

$$
\left(I-H H^{\dagger}\right) f(x)=0, \quad \text { then } f_{1}(x)=f_{4}(x), \quad 0 \leqslant x \leqslant 1 .
$$

Projections $g_{l}(x)$ defined by $(3.26)$ are

$$
\begin{aligned}
& g_{1}(x)=\left[\begin{array}{lll}
H(0,2) & 0 & 0
\end{array}\right] H^{\dagger} f(x)=\left(\begin{array}{llll}
0 & f_{2}(x) & 0 & 0
\end{array}\right)^{\mathrm{T}} \\
& g_{2}(x)=\left[\begin{array}{lllll}
0 & H(0,-1) & 0
\end{array}\right] H^{\dagger} f(x)=\left(\begin{array}{llll}
f_{1}(x) & 0 & f_{1}(x) & f_{1}(x)
\end{array}\right)^{\mathrm{T}} \\
& g_{3}(x)=\left[\begin{array}{lll}
0 & 0 & H(2,1)
\end{array}\right] H^{\dagger} f(x)=\left(\begin{array}{llll}
0 & 0 & f_{3}(x) f_{1}(x) & 0
\end{array}\right)^{\mathrm{T}} .
\end{aligned}
$$

Since

$$
\begin{gathered}
{[H(0,2)]^{\dagger}=\left[\begin{array}{cccc}
0 & 0 & 0 & 0 \\
0 & 1 & 0 & 0 \\
0 & 0 & 0 & 0 \\
0 & 0 & 0 & 0
\end{array}\right], \quad[H(0,-1)]^{\dagger}=\frac{1}{3}\left[\begin{array}{cccc}
1 & 0 & 1 & 1 \\
0 & 0 & 0 & 0 \\
1 & 0 & 1 & 1 \\
1 & 0 & 1 & 1
\end{array}\right],} \\
{[H(2,1)]^{\dagger}=\left[\begin{array}{llll}
0 & 0 & 0 & 0 \\
0 & 0 & 0 & 0 \\
0 & 0 & 1 & 0 \\
0 & 0 & 0 & 0
\end{array}\right],}
\end{gathered}
$$

and

$$
\begin{aligned}
{\left[I-H(0,2)[H(0,2)]^{\dagger}\right] A H(0,2) } & =0, \\
{\left[I-H(0,-1)[H(0,-1)]^{\dagger}\right] A H(0,-1) } & =0, \\
{\left[I-H(2,1)[H(2,1)]^{\dagger}\right] A H(2,1) } & =0,
\end{aligned}
$$

Thus, condition (3.30) holds true and the subspaces $\operatorname{Im} H(0,2), \operatorname{Im} H(0,-1)$, and also $\operatorname{Im} H(2,1)$, are invariant by the stable matrix $A$. Well-posedness conditions (3.24) take the form

$$
\begin{gathered}
{\left[\begin{array}{llll}
0 & 0 & 0 & 0 \\
0 & 1 & 0 & 0 \\
0 & 0 & 0 & 0 \\
0 & 0 & 0 & 0
\end{array}\right]\left(f(0)+2 f^{\prime}(0)\right)=0 \text { or } f_{2}(0)+2 f_{2}^{\prime}(0)=0} \\
{\left[\begin{array}{llll}
0 & 0 & 0 & 0 \\
0 & 1 & 0 & 0 \\
0 & 0 & 0 & 0 \\
0 & 0 & 0 & 0
\end{array}\right] f^{\prime}(1)=0 \quad \text { or } \quad f_{2}^{\prime}(1)=0}
\end{gathered}
$$




$$
\begin{aligned}
& \frac{1}{2}\left[\begin{array}{llll}
1 & 0 & 0 & 1 \\
0 & 0 & 0 & 0 \\
1 & 0 & 0 & 1 \\
1 & 0 & 0 & 1
\end{array}\right]\left(f(0)-f^{\prime}(0)\right)=0 \text { or } f_{1}(0)-f_{1}^{\prime}(0)=0, \\
& \frac{1}{2}\left[\begin{array}{llll}
1 & 0 & 0 & 1 \\
0 & 0 & 0 & 0 \\
1 & 0 & 0 & 1 \\
1 & 0 & 0 & 1
\end{array}\right] f^{\prime}(1)=0 \quad \text { or } \quad f_{1}^{\prime}(1)=0 \\
& \frac{1}{2}\left[\begin{array}{rrrr}
0 & 0 & 0 & 0 \\
0 & 0 & 0 & 0 \\
-1 & 0 & 2 & -1 \\
0 & 0 & 0 & 0
\end{array}\right]\left(f(0)+f^{\prime}(0)\right)=0 \quad \text { or } \quad f_{3}(0)-f_{1}(0)+f_{3}^{\prime}(0)-f_{1}^{\prime}(0)=0 \text {, } \\
& \frac{1}{2}\left[\begin{array}{rrrr}
0 & 0 & 0 & 0 \\
0 & 0 & 0 & 0 \\
-1 & 0 & 2 & -1 \\
0 & 0 & 0 & 0
\end{array}\right]\left(2 f(1)+f^{\prime}(1)\right)=0 \quad \text { or } \quad 2 f_{3}(1)-2 f_{1}(1)+f_{3}^{\prime}(1)-f_{1}^{\prime}(1)=0 \text {. }
\end{aligned}
$$

Summarizing the corresponding problems (1.1)-(1.4) is well-posed and satisfies hypotheses of Theorem 3.3 if $f(x)$ is twice continuously differentiable in $[0,1]$ and verifies the conditions

$$
\begin{aligned}
& f_{1}(x)=f_{4}(x), \quad 0 \leqslant x \leqslant 1 \\
& f_{1}(0)-f_{1}^{\prime}(0)=0, f_{2}(0)+2 f_{2}^{\prime}(0)=0, \\
& f_{1}^{\prime}(1)=0, f_{2}^{\prime}(1)=0, \quad 2 f_{3}(1)+f_{3}^{\prime}(1)=2 f_{1}(1) .
\end{aligned}
$$

\section{Analytic-numerical solutions with prefixed accuracy}

The series solution of problems (1.1)-(1.4) provided by Theorem 3.3 presents some computational difficulties. Firstly, the infiniteness of the series. Secondly, eigenvalues are not exactly computable because equation (2.15) is not solvable in a closed form. It is important to point out here that eigenvalues of the coupled problems (1.1)-(1.4) and eigenfunctions are built up in terms of scalar Sturm-Liouville problems of the type (3.7) or (3.29). In spite of well-known efficient numerical algorithms for the computations of eigenvalues $[\mathbf{1 8}, \mathbf{2 4}, \mathbf{2 5}]$, it is interesting to study the admissible tolerance in the approximate eigenvalues according with a prefixed accuracy. Finally, as the computation of matrix exponentials appearing in the exact solution of problems (1.1)-(1.4) is not an easy task (see [21]), we also approximate matrix exponentials by appropriate matrix polynomials of certain degree. In this section we address the following question. Given an admissible error $\varepsilon>0$ and a bounded subdomain $D\left(t_{0}, t_{1}\right)=\left\{(x, t) ; 0 \leqslant x \leqslant 1,0 \leqslant t_{0} \leqslant t \leqslant t_{1}\right\}$, how do we construct an approximation that avoids the above-quoted difficulties and whose error with respect to the exact solution is less than $\varepsilon$ uniformly in $D\left(t_{0}, t_{1}\right)$. By 
Theorem 3.3 it is sufficient to develop the approach when the exact series solution is the given by Theorem 3.1 .

To fix ideas we seek to approximate the series $U(x, t)$ defined by $(3.11)$, where vector $E_{\lambda_{n}}$ is given by (3.9)-(3.10). By applying Parseval's inequality (see [3, p. 223] and [7]) to the scalar Sturm-Liouville problem (3.7), one gets

$$
\begin{gathered}
\left|e_{\lambda_{n}}(i)\right|^{2} \leqslant \int_{0}^{1}\left|f_{i}(x)\right|^{2} \mathrm{~d} x, \quad n \geqslant 1, \quad 1 \leqslant i \leqslant m, \\
\left\|E_{\lambda_{n}}\right\|^{2} \leqslant \sum_{j=1}^{m} \int_{0}^{1}\left|f_{j}(x)\right|^{2} \mathrm{~d} x=\int_{0}^{1}\|f(x)\|_{2}^{2} \mathrm{~d} x=F^{2}, \quad n \geqslant 1 .
\end{gathered}
$$

By Theorem 3.1 we have $\beta \in \sigma\left(B_{1}\right)$, and, by (3.17), one gets $B_{1}\left(E_{\lambda_{n}}\right)=\beta E_{\lambda_{n}}$ and

$$
X_{\lambda_{n}}(x)=\left\{\sin \left(\lambda_{n} x\right)-\lambda_{n} \beta \cos \left(\lambda_{n} x\right)\right\} E_{\lambda_{n}}, \quad n \geqslant 1, \quad 0 \leqslant x \leqslant 1 .
$$

By (3.32)-(4.3), it follows that

$$
\left\|X_{\lambda_{n}}(x)\right\| \leqslant F\left(1+\lambda_{n}\left\|B_{1}\right\|\right), \quad 0 \leqslant x \leqslant 1, \quad n \geqslant 1 .
$$

By $(1.8)$ for $t_{1} \geqslant t \geqslant t_{0}$, one gets

$$
\left\|\mathrm{e}^{-\lambda_{n}^{2} A t}\right\| \leqslant \mathrm{e}^{-\beta(A) t_{0} \lambda_{n}^{2}} \sum_{j=0}^{m-1} \frac{\left(\|A\| t_{1} \sqrt{m}\right)^{j}}{j !} \lambda_{n}^{2 j}
$$

Let $\varphi_{k}$ and $\phi_{k}$ be the scalar functions defined for $s>0$ by

$$
\varphi_{k}(s)=(k+2) \ln (s)-s^{2} \beta(A) t_{0}, \quad \phi_{k}(s)=\mathrm{e}^{-s^{2} \beta(A) t_{0}} s^{k}, \quad 0 \leqslant k \leqslant 2 m-1 .
$$

Since

$$
\varphi_{k}^{\prime}(s)=\frac{(k+2)}{s}-2 s \beta(A) t_{0}
$$

it follows that

$$
\varphi_{k}^{\prime}(s)<0, \quad \text { if } s>s_{k}=\left(\frac{k+2}{\beta(A) t_{0}}\right)^{1 / 2}, \quad 0 \leqslant k \leqslant 2 m-1 .
$$

Take $s_{k}^{\prime} \geqslant s_{k}$ such that

$$
(k+2) \ln (s)-s^{2} \beta(A) t_{0}<0, \quad s \geqslant s_{k}^{\prime} \geqslant s_{k}, \quad 0 \leqslant k \leqslant 2 m-1,
$$

then, by (4.7), it follows that

$$
\phi_{k}(s)=\mathrm{e}^{-s^{2} \beta(A) t_{0}} s^{k}<\left(1+\left\|B_{1}\right\|\right)^{-1} s^{-2}, \quad s \geqslant s_{k}^{\prime}, \quad 0 \leqslant k \leqslant 2 m-1 .
$$

Since $\lim _{n \rightarrow \infty} \lambda_{n}=+\infty$ and $\lambda_{n}<\lambda_{n+1}$, let $n_{0}$ be the first positive integer so that

$$
\lambda_{n_{0}}>s^{*}=\max \left\{s_{k}^{\prime} ; 0 \leqslant k \leqslant 2 m-1\right\} \text {. }
$$


By (4.3)-(4.9), it follows that

$$
\begin{aligned}
\left\|\mathrm{e}^{-\lambda_{n}^{2} A t} X_{\lambda_{n}}(x)\right\| & \leqslant F \sum_{j=0}^{m-1} \frac{\left(\phi_{2 j}\left(\lambda_{n}\right)+\left\|B_{1}\right\| \phi_{2 j+1}\left(\lambda_{n}\right)\right)}{j !}\left(\|A\| t_{1} \sqrt{m}\right)^{j} \\
& \leqslant \lambda_{n}^{-2}\left(\sum_{j=0}^{m-1} \frac{\left(\|A\| t_{1} \sqrt{m}\right)^{j}}{j !}\right), \quad 0 \leqslant x \leqslant 1, \quad 0<t_{0} \leqslant t \leqslant t_{1}, \quad n \geqslant n_{0} .
\end{aligned}
$$

Since $\lambda_{n}>(n-1) \pi, n \geqslant 1$ (see Remark 2.2), if we denote by $L$ the constant

$$
L=F\left(\sum_{j=0}^{m-1} \frac{\left(\|A\| t_{1} \sqrt{m}\right)^{j}}{j !}\right)
$$

then

$$
\left\|\sum_{n>n_{0}} \mathrm{e}^{-\lambda_{n}^{2} A t} X_{\lambda_{n}}(x)\right\|_{2} \leqslant L \sum_{n>n_{0}} \lambda_{n}^{-2} \leqslant \frac{L}{\pi^{2}} \sum_{n>n_{0}} n^{-2} .
$$

Since $\sum_{n \geqslant 1} n^{-2}=\frac{1}{6} \pi^{2}$, taking $n_{1}>n_{0}$ so that

$$
\sum_{n=1}^{n_{1}} n^{-2}>\pi^{2}\left(\frac{1}{6}-\frac{\varepsilon}{3 L}\right)
$$

by (4.11) and (4.12), one gets

$$
\left\|\sum_{n>n_{1}} \mathrm{e}^{-\lambda_{n}^{2} A t} X_{\lambda_{n}}(x)\right\|_{2} \leqslant \frac{1}{3} \varepsilon, \quad 0 \leqslant x \leqslant 1, \quad 0<t_{0} \leqslant t .
$$

Thus, the finite sum

$$
V\left(x, t, n_{1}\right)=\sum_{n=1}^{n_{1}} \mathrm{e}^{-\lambda_{n}^{2} A t} X_{\lambda_{n}}(x)
$$

satisfies

$$
\left\|U(x, t)-V\left(x, t, n_{1}\right)\right\|_{2}<\frac{1}{3} \varepsilon, \quad 0 \leqslant x \leqslant 1, \quad 0<t_{0} \leqslant t .
$$

The approximation $V\left(x, t, n_{1}\right)$ involves computation of the exact eigenvalues $\lambda_{1}, \lambda_{2}, \ldots$, $\lambda_{n_{1}}$, which is not easy in practice. Now we study the admissible tolerance when one considers approximate eigenvalues $\tilde{\lambda}_{1}, \tilde{\lambda}_{2}, \ldots, \tilde{\lambda}_{n_{1}}$, building up the approximation of $V\left(x, t, n_{1}\right)$ defined by

$$
\left.\begin{array}{c}
\tilde{V}\left(x, t, n_{1}\right)=\sum_{n=1}^{n_{1}} \mathrm{e}^{-\tilde{\lambda}_{n}^{2} A t} X_{\tilde{\lambda}_{n}}(x), \\
X_{\tilde{\lambda}_{n}}(x)=\left\{\sin \left(\tilde{\lambda}_{n} x\right)-\tilde{\lambda}_{n} \beta \cos \left(\tilde{\lambda}_{n} x\right)\right\} E_{\tilde{\lambda}_{n}}, \quad E_{\tilde{\lambda}_{n}}=\left[\begin{array}{c}
e_{\tilde{\lambda}_{n}}(1) \\
\vdots \\
e_{\tilde{\lambda}_{n}}(m)
\end{array}\right],
\end{array}\right\}
$$


where, for $1 \leqslant i \leqslant m, e_{\tilde{\lambda}_{n}}(i)$ is defined replacing $\lambda_{n}$ by $\tilde{\lambda}_{n}$ in (3.9). Note that we can write

$$
\begin{aligned}
\mathrm{e}^{-\tilde{\lambda}_{n}^{2} A t} X_{\tilde{\lambda}_{n}}(x),-\mathrm{e}^{-\lambda_{n}^{2} A t} X_{\lambda_{n}}(x) \\
=\mathrm{e}^{-\bar{\lambda}_{n}^{2} A t}\left\{\sin \left(\tilde{\lambda}_{n} x\right)-\tilde{\lambda}_{n} \beta \cos \left(\tilde{\lambda}_{n} x\right)\right\} E_{\tilde{\lambda}_{n}}-\mathrm{e}^{-\lambda_{n}^{2} A t}\left\{\sin \left(\lambda_{n} x\right)-\lambda_{n} \beta \cos \left(\lambda_{n} x\right)\right\} E_{\lambda_{n}} \\
=\left(\mathrm{e}^{-\tilde{\lambda}_{n}^{2} A t}-\mathrm{e}^{-\lambda_{n}^{2} A t}\right)\left\{\sin \left(\tilde{\lambda}_{n} x\right)-\tilde{\lambda}_{n} \beta \cos \left(\tilde{\lambda}_{n} x\right)\right\} E_{\tilde{\lambda}_{n}} \\
\quad+\mathrm{e}^{-\tilde{\lambda}_{n}^{2} A t}\left\{\sin \left(\tilde{\lambda}_{n} x\right)-\tilde{\lambda}_{n} \beta \cos \left(\tilde{\lambda}_{n} x\right)-\sin \left(\lambda_{n} x\right)+\lambda_{n} \beta \cos \left(\lambda_{n} x\right)\right\} E_{\tilde{\lambda}_{n}} \\
\quad+\mathrm{e}^{-\lambda_{n}^{2} A t}\left\{\sin \left(\lambda_{n} x\right)-\lambda_{n} \beta \cos \left(\lambda_{n} x\right)\right\}\left(E_{\tilde{\lambda}_{n}}-E_{\lambda_{n}}\right) .
\end{aligned}
$$

Let $I(\rho)$ be defined by

$$
I(\rho)=\int_{0}^{1}\{\sin (\rho x)-\beta \rho \cos (\rho x)\}^{2} \mathrm{~d} x, \quad \rho>0
$$

and let $\gamma, \Lambda$ and $\Lambda_{1}$ be positive constants chosen so that

$$
\left.\begin{array}{c}
\inf \left\{I(\rho) ; \rho=\lambda_{n}, \rho=\tilde{\lambda}_{n}, 1 \leqslant n \leqslant n_{1}\right\} \geqslant \gamma^{-1} \\
0<\Lambda_{1}<\min \left\{\lambda_{1}, \tilde{\lambda}_{1}\right\}, \quad \max \left\{\lambda_{n}, \tilde{\lambda}_{n} ; 1 \leqslant n \leqslant n_{1}\right\} \leqslant \Lambda .
\end{array}\right\}
$$

It is easy to show that

$$
\left.\begin{array}{c}
\left|\sin \left(\tilde{\lambda}_{n} x\right)-\tilde{\lambda}_{n} \beta \cos \left(\tilde{\lambda}_{n} x\right)-\sin \left(\lambda_{n} x\right)+\lambda_{n} \beta \cos \left(\lambda_{n} x\right)\right| \leqslant\left(1+\beta|+| \beta \mid \lambda_{n}\right)\left|\lambda_{n}-\tilde{\lambda}_{n}\right|, \\
\left|\sin \left(\tilde{\lambda}_{n} x\right)-\tilde{\lambda}_{n} \beta \cos \left(\tilde{\lambda}_{n} x\right)\right| \leqslant 1+\left|\tilde{\lambda}_{n}\right|, \quad 0 \leqslant x \leqslant 1 .
\end{array}\right\}
$$

By $(3.9)$, for $1 \leqslant i \leqslant m$, one gets

$$
\begin{aligned}
e_{\lambda_{n}}(i)-e_{\tilde{\lambda}_{n}}(i)= & \frac{\left(I\left(\lambda_{n}\right)-I\left(\tilde{\lambda}_{n}\right)\right) \int_{0}^{1} f_{i}(x)\left\{\sin \left(\tilde{\lambda}_{n} x\right)-\tilde{\lambda}_{n} \beta \cos \left(\tilde{\lambda}_{n} x\right)\right\} \mathrm{d} x}{I\left(\lambda_{n}\right) I\left(\tilde{\lambda}_{n}\right)} \\
& +\frac{\int_{0}^{1} f_{i}(x)\left\{\sin \left(\lambda_{n} x\right)-\lambda_{n} \beta \cos \left(\lambda_{n} x\right)-\sin \left(\tilde{\lambda}_{n} x\right)+\tilde{\lambda}_{n} \beta \cos \left(\tilde{\lambda}_{n} x\right)\right\} \mathrm{d} x}{I\left(\lambda_{n}\right)}
\end{aligned}
$$

by the Cauchy-Schwarz inequality for integrals it follows that

$$
\int_{0}^{1}\left|f_{i}(x)\left\{\sin \left(\tilde{\lambda}_{n} x\right)-\tilde{\lambda}_{n} \beta \cos \left(\tilde{\lambda}_{n} x\right)\right\}\right| \mathrm{d} x \leqslant\left(\int_{0}^{1}\left|f_{i}(x)\right|^{2} \mathrm{~d} x\right)^{1 / 2}\left(I\left(\tilde{\lambda}_{n}\right)\right)^{1 / 2}
$$

and by (4.19)

$$
\begin{aligned}
& \int_{0}^{1}\left|f_{i}(x)\left\{\sin \left(\lambda_{n} x\right)-\lambda_{n} \beta \cos \left(\lambda_{n} x\right)-\sin \left(\tilde{\lambda}_{n} x\right)+\tilde{\lambda}_{n} \beta \cos \left(\tilde{\lambda}_{n} x\right)\right\}\right| \mathrm{d} x \\
& \leqslant\left(1+\left\|B_{1}\right\|\left(1+\lambda_{n}\right)\right)\left(\int_{0}^{1}\left|f_{i}(x)\right|^{2} \mathrm{~d} x\right)^{1 / 2}\left|\lambda_{n}-\tilde{\lambda}_{n}\right|, \quad 1 \leqslant i \leqslant m .
\end{aligned}
$$


By (4.20)-(4.22) it follows that

$$
\left|e_{\lambda_{n}}(i)-e_{\tilde{\lambda}_{n}}(i)\right| \leqslant\left\{\frac{\left|I\left(\lambda_{n}\right)-I\left(\tilde{\lambda}_{n}\right)\right|}{\left(I\left(\tilde{\lambda}_{n}\right)\right)^{1 / 2}}+\left|\lambda_{n}-\tilde{\lambda}_{n}\right|\left(1+\left\|B_{1}\right\|\left(1+\lambda_{n}\right)\right)\right\} \frac{\left(\int_{0}^{1}\left|f_{i}(x)\right|^{2} \mathrm{~d} x\right)^{1 / 2}}{I\left(\lambda_{n}\right)}
$$

Note that

$$
\begin{aligned}
& I\left(\lambda_{n}\right)-I\left(\tilde{\lambda}_{n}\right)=\int_{0}^{1}\left(\sin \left(\lambda_{n} x\right)\right.\left.-\lambda_{n} \beta \cos \left(\lambda_{n} x\right)+\sin \left(\tilde{\lambda}_{n} x\right)-\tilde{\lambda}_{n} \beta \cos \left(\tilde{\lambda}_{n} x\right)\right) \\
& \times\left(\sin \left(\lambda_{n} x\right)-\lambda_{n} \beta \cos \left(\lambda_{n} x\right)-\sin \left(\tilde{\lambda}_{n} x\right)+\tilde{\lambda}_{n} \beta \cos \left(\tilde{\lambda}_{n} x\right)\right) \mathrm{d} x
\end{aligned}
$$

and by (4.18) and (4.19) one gets

$$
\begin{aligned}
\left|I\left(\lambda_{n}\right)-I\left(\tilde{\lambda}_{n}\right)\right| \leqslant\left|\lambda_{n}-\tilde{\lambda}_{n}\right|\left(1+\left\|B_{1}\right\|\left(1+\lambda_{n}+\tilde{\lambda}_{n}\right)\right)^{2} & \\
\left|e_{\lambda_{n}}(i)-e_{\bar{\lambda}_{n}}(i)\right| \leqslant\left(1+\left(I\left(\lambda_{n}\right)\right)^{-1 / 2}\right)\left(I\left(\lambda_{n}\right)\right)^{-1}\left(1+\left\|B_{1}\right\|\left(1+\lambda_{n}+\tilde{\lambda}_{n}\right)\right)^{2} & \times\left(\int_{0}^{1}\left|f_{i}(x)\right|^{2} \mathrm{~d} x\right)^{1 / 2}\left|\lambda_{n}-\tilde{\lambda}_{n}\right|, \\
\left|e_{\lambda_{n}}(i)-e_{\bar{\lambda}_{n}}(i)\right| \leqslant 4\left(\gamma^{1 / 2}+\right. & 1) \gamma\left(1+\left\|B_{1}\right\|\right)^{2}(1+\Lambda) \\
& \times\left(\int_{0}^{1}\left|f_{i}(x)\right|^{2} \mathrm{~d} x\right)^{1 / 2}\left|\lambda_{n}-\tilde{\lambda}_{n}\right|, \quad 1 \leqslant n \leqslant n_{1}, \\
\left\|E_{\lambda_{n}}-E_{\tilde{\lambda}_{n}}\right\|_{2} \leqslant 4\left(\gamma^{1 / 2}\right. & +1) \gamma\left(1+\left\|B_{1}\right\|\right)^{2}(1+\Lambda) \\
& \times\left(\int_{0}^{1}\left\|f_{i}(x)\right\|_{2}^{2} \mathrm{~d} x\right)^{1 / 2}\left|\lambda_{n}-\tilde{\lambda}_{n}\right|, \quad 1 \leqslant n \leqslant n_{1} .
\end{aligned}
$$

By definition of $E_{\tilde{\lambda}_{n}}$ we have

$$
\left\|E_{\tilde{\lambda}_{n}}\right\|_{2} \leqslant 2\left(1+\tilde{\lambda}_{n}\left\|B_{1}\right\|\right)\left(\int_{0}^{1}\left\|f_{i}(x)\right\|_{2}^{2} \mathrm{~d} x\right)^{1 / 2}, \quad 1 \leqslant n \leqslant n_{1} .
$$

By (1.8) and (4.18) one gets

$$
\left.\begin{array}{l}
\left\|\mathrm{e}^{-\lambda_{n}^{2} A t}\right\| \leqslant \mathrm{e}^{-t_{0} \beta(A) \Lambda_{1}^{2}} \sum_{j=0}^{m-1} \frac{\left(\Lambda^{2} t_{1}\|A\| \sqrt{m}\right)^{j}}{j !}, \\
\left\|\mathrm{e}^{-\bar{\lambda}_{n}^{2} A t}\right\| \leqslant \mathrm{e}^{-t_{0} \beta(A) \Lambda_{1}^{2}} \sum_{j=0}^{m-1} \frac{\left(\Lambda^{2} t_{1}\|A\| \sqrt{m}\right)^{j}}{j !}, \quad 1 \leqslant n \leqslant n_{1}, \quad t_{0} \leqslant t \leqslant t_{1} .
\end{array}\right\}
$$

Let us write

$$
\mathrm{e}^{-t \lambda_{n}^{2} A}-\mathrm{e}^{-t \bar{\lambda}_{n}^{2} A}=\mathrm{e}^{-t \bar{\lambda}_{n}^{2} A}\left(\mathrm{e}^{-t\left(\lambda_{n}^{2}-\tilde{\lambda}_{n}^{2}\right) A}-I\right)
$$


By (1.8), (4.18) and the mean value theorem, under the hypothesis $\left|\lambda_{n}-\bar{\lambda}_{n}\right|<1$ one gets

$$
\begin{aligned}
& \left\|\mathrm{e}^{-t \lambda_{n}^{2} A}-\mathrm{e}^{-t \bar{\lambda}_{n}^{2} A}\right\| \leqslant\left\|\mathrm{e}^{-t \bar{\lambda}_{n}^{2} A}\right\|\left(\mathrm{e}^{-t\left(\lambda_{n}^{2}-\tilde{\lambda}_{n}^{2}\right)\|A\|}-1\right), \quad 1 \leqslant n \leqslant n_{1}, \\
& \left\|\mathrm{e}^{-t \lambda_{n}^{2} A}-\mathrm{e}^{-t \bar{\lambda}_{n}^{2} A}\right\| \leqslant\left(\mathrm{e}^{-t_{0} \beta(A) \Lambda_{1}^{2}}\left[\sum_{j=0}^{m-1} \frac{\left(\Lambda^{2} t_{1}\|A\| \sqrt{m}\right)^{j}}{j !}\right] 4 \Lambda\|A\| t_{1} \mathrm{e}^{2\|A\| \Lambda t_{1}}\right)\left|\lambda_{n}-\tilde{\lambda}_{n}\right| .
\end{aligned}
$$

By (4.16), (4.23), (4.24), (4.25) and (4.26), assuming that $\left|\lambda_{n}-\tilde{\lambda}_{n}\right|<1,1 \leqslant n \leqslant n_{1}$, $t_{0} \leqslant t \leqslant t_{1}$, it follows that

$$
\left\|\mathrm{e}^{-t \tilde{\lambda}_{n}^{2} A} X_{\bar{\lambda}_{n}}(x)-\mathrm{e}^{-t \lambda_{n}^{2} A} X_{\lambda_{n}}(x)\right\|_{2} \leqslant K\left|\lambda_{n}-\tilde{\lambda}_{n}\right|, \quad 1 \leqslant n \leqslant n_{1}, \quad t_{0} \leqslant t \leqslant t_{1},
$$

where

$$
\left.\begin{array}{rl}
K & =4\left[\sum_{j=0}^{m-1} \frac{\left(\Lambda^{2} t_{1}\|A\| \sqrt{m}\right)^{j}}{j !}\right](1+\Lambda)^{2} \mathrm{e}^{-t_{0} \beta(A) \Lambda_{1}^{2}} K_{1}, \\
K_{1} & =\left(\gamma^{1 / 2}+1\right) \gamma\left(1+\left\|B_{1}\right\|\right)^{2}+\|A\| t_{1} \mathrm{e}^{2\|A\| \Lambda t_{1}}+\left(\int_{0}^{1}\left\|f_{i}(x)\right\|_{2}^{2} \mathrm{~d} x\right)^{1 / 2} \cdot
\end{array}\right\}
$$

Given $\varepsilon>0$ and $n_{1}$, consider approximations $\tilde{\lambda}_{n}$ of $\lambda_{n}$ for $1 \leqslant n \leqslant n_{1}$, so that

$$
\left|\lambda_{n}-\tilde{\lambda}_{n}\right|<\min \left(1, \frac{\varepsilon}{3 n_{1} K}\right), \quad 1 \leqslant n \leqslant n_{1}
$$

then by (4.14), (4.16), (4.27) and (4.28) it follows that

$$
\left\|V\left(x, t, n_{1}\right)-\tilde{V}\left(x, t, n_{1}\right)\right\|_{2}<\frac{1}{3} \varepsilon, \quad t_{0} \leqslant t \leqslant t_{1}, \quad 0 \leqslant x \leqslant 1 .
$$

By Theorem 11.2 .4 of $[11$, p. 550], one gets

$$
\left\|\mathrm{e}^{-t \tilde{\lambda}_{n}^{2} A}-\sum_{k=0}^{q} \frac{\left(-\tilde{\lambda}_{n}^{2} t A\right)^{k}}{k !}\right\| \leqslant \frac{m}{(q+1) !}\left(\tilde{\lambda}_{n}^{2} t_{1}\|A\|\right)^{q+1} \mathrm{e}^{t_{1} \tilde{\lambda}_{n}^{2}\|A\|}, \quad t_{0} \leqslant t \leqslant t_{1}
$$

and by (4.18) and (4.19),

$$
\left\|X_{\tilde{\lambda}_{n}}(x)\right\|_{2} \leqslant 2\left(1+\Lambda\left\|B_{1}\right\|\right)^{2}\left(\int_{0}^{1}\|f(x)\|_{2}^{2} \mathrm{~d} x\right)^{1 / 2}, \quad 1 \leqslant n \leqslant n_{1}, \quad 0 \leqslant x \leqslant 1 .
$$

Since

$$
\lim _{q \rightarrow \infty} \frac{\left(\Lambda^{2} t_{1}\|A\|\right)^{q+1}}{(q+1) !}=0
$$

take the first positive integer $q_{0}$ such that

$$
\frac{\left(\Lambda^{2} t_{1}\|A\|\right)^{q_{0}+1}}{\left(q_{0}+1\right) !}<\frac{\varepsilon}{6 n_{1} \mathrm{e}^{\|A\| A^{2} t_{1}}\left(1+\Lambda\left\|B_{1}\right\|\right)^{2}\left(\int_{0}^{1}\|f(x)\|_{2}^{2} \mathrm{~d} x\right)^{1 / 2}}
$$


then, if we define

$$
\tilde{u}\left(x, t, n_{1}, q_{0}\right)=\sum_{n=1}^{n_{1}} \sum_{k=0}^{q_{0}} \frac{\left(-\tilde{\lambda}_{n}^{2} t A\right)^{k}}{k !} X_{\tilde{\lambda}_{n}}(x),
$$

by (4.16), (4.31) and (4.32) one gets

$$
\left\|\tilde{V}\left(x, t, n_{1}\right)-\tilde{u}\left(x, t, n_{1}, q_{0}\right)\right\|_{2}<\frac{1}{3} \varepsilon, \quad t_{0} \leqslant t \leqslant t_{1}, \quad 0 \leqslant x \leqslant 1,
$$

and by (4.15), (4.29) and (4.33) one concludes that

$$
\left\|U(x, t)-\tilde{u}\left(x, t, n_{1}, q_{0}\right)\right\|_{2}<\varepsilon, \quad t_{0} \leqslant t \leqslant t_{1}, \quad 0 \leqslant x \leqslant 1 .
$$

Summarizing, the following result has been established.

Theorem 4.1. With the hypotheses and the notation of Theorem 3.1, let $\varepsilon>0$, $t_{0}>0$ and $D\left(t_{0}, t_{1}\right)=\left\{(x, t) ; 0 \leqslant x \leqslant 1, t_{0} \leqslant t \leqslant t_{1}\right\}$. Let $\gamma, \Lambda$ and $\Lambda_{1}$ be defined by (4.18). Let $n_{1}$ be chosen by (4.12) and $q_{0}$ by (4.31). Let $\tilde{\lambda}_{1}, \tilde{\lambda}_{2}, \ldots, \tilde{\lambda}_{n_{1}}$ be approximations of eigenvalues $\lambda_{1}, \lambda_{2}, \ldots, \lambda_{n_{1}}$ satisfying

$$
\left|\lambda_{n}-\tilde{\lambda}_{n}\right|<\min \left(1, \frac{\varepsilon}{3 n_{1} K}\right), \quad 1 \leqslant n \leqslant n_{1},
$$

where $K$ is given by (4.28). Then $\tilde{u}\left(x, t, n_{1}, q_{0}\right)$, defined by (4.32), is an approximation of the exact solution $U(x, t)$ of problems (1.1)-(1.4), given by Theorem 3.1, satisfying (4.34).

Acknowledgements. This work has been partly supported by the Spanish DGICYT grant PB96-1321-CO2-02 and the Generalitat Valenciana grants GV-C-CN-1005796 and GV-97-CB-1263.

\section{References}

1. M. H. Alexander And D. E. Manolopoulos, A stable linear reference potencial algorithm for solution of the quantum close-coupled equations in molecular scattering theory, J. Chem. Phys. 86 (1987), 2044-2050.

2. T. M. APostol, Mathematical analysis (Addison-Wesley, Reading, MA, 1977).

3. F. V. ATKINSON, Discrete and continuous boundary value problems (Academic, New York, 1964).

4. F. V. Atkinson, A. M. Krall, G. K. Leaf and A. Zettel, On the numerical computation of eigenvalues of Sturm-Liouville problems with matrix coefficients, Technical Report, Argonne National Laboratory, 1987.

5. O. AXElsson, Iterative solution methods (Cambridge University Press, 1994).

6. S. L. CAMPBell AND C. D. MeYer JR, Generalized inverses of linear transformations (Pitman, London, 1979).

7. E. A. CODDINGTON AND N. LEvinson, Theory of ordinary differential equations (McGraw-Hill, New York, 1967).

8. J. CRANK, The mathematics of diffusion, 2nd edn (Oxford University Press, 1995).

9. N. DUNFORD AND J. SchWARTZ, Linear operators, part I (Interscience, New York, 1957). 
10. G. B. Folland, Fourier analysis and its applications (Wadworth \& Brooks, Pacific Grove, CA, 1992).

11. G. H. Golub and C. F. VAN LOAN, Matrix computation (The Johns Hopkins University Press, Baltimore, MD, 1989).

12. L. Greenberg, A Prüfer method for calculating eigenvalues of self-adjoint systems of ordinary differential equations, parts 1 and 2, University of Maryland, Technical Report TR91-24.

13. T. Hueckel, M. Borsetto and A. Peano, Modelling of coupled thermo-elastoplastic hydraulic response of clays subjected to nuclear waste heat, in Numerical methods in transient and coupled problems (ed. R. W. Lewis, E. Hinton, P. Bettes and B. A. Scherefler), pp. 213-235 (Wiley, New York, 1987).

14. E. L. INCE, Ordinary differential equations (Dover, New York, 1927).

15. L. JÓDAR AND E. PONSODA, Continuous numerical solutionand error bounds for time dependent systems of partial differential equations: mixed problems, Comp. Math. Appl. 29 (1995), 63-71.

16. R. D. Levine, M. Shapiro And B. Johnson, Transition probabilities in molecular collisions: computational studies of rotational excitation, J. Chem. Phys. 53 (1970), 17551766 .

17. J. V. Lill, T. G. Schmalz and J. C. Light, Imbedded matrix Green's functions in atomic and molecular scattering theory, J. Chem. Phys. 78 (1983), 4456-4463.

18. M. MARLETTA, Theory and implementation of algorithms for Sturm-Liouville systems, PhD thesis, Royal Military College of Science, Cranfield, 1991.

19. V. S. MelezhiK, I. V. Puzynin, T. P. PuzYnina AND L. N. Somov, Numerical solution of a system of integrodifferential equations arising from the quantum-mechanical threebody problem with Coulomb interaction, J. Comput. Phys. 54 (1984), 221-236.

20. M. D. MIKhaILOV AND M. N. ÖsIZIK, Unifield analysis and solutions of heat and mass diffusion (Wiley, New York, 1984).

21. C. B. MOLER AND C. F. VAN LOAN, Nineteen dubious ways to compute the exponential of a matrix, SIAM Rev. 20 (1978), 801-836.

22. F. Mrugala and D. Secrest, The generalized log-derivate method for inelastic and reactive collisions, J. Chem. Phys. 78 (1983), 5954-5961.

23. E. NAVARRO, E. PONSODA AND L. JóDAR, A matrix approach to the analytic-numerical solution of mixed partial differential systems, Comp. Math. Appl. 30 (1995), 99-109.

24. J. D. PRYCE, Numerical solution of Sturm-Liouville problems (Clarendon, Oxford, 1993).

25. J. D. PRYCe AND M. MarletTa, Automatic solution of Sturm-Liouville problems using Pruess method, J. Comput. Appl. Math. 39 (1992), 57-78.

26. C. R. RAO AND S. K. Mitra, Generalized inverse of matrices and its applications (Wiley, New York, 1971).

27. W. T. REID, Ordinary differential equations (Wiley, New York, 1971).

28. M. Shapiro AND G. G. Balint-KurTi, A new method for the exact calculation of vibrational-rotational energy levels of triatomic molecules, J. Chem. Phys. 71 (1979), 1461-1469.

29. I. STAKGOLD, Green's functions and boundary value problems (Wiley, New York, 1979). 